\title{
A stabilized assumed deformation gradient finite element formulation for strongly coupled poromechanical simulations at finite strain
}

\author{
WaiChing Sun ${ }^{1, *, \dagger}$, Jakob T. Ostien ${ }^{1}$ and Andrew G. Salinger ${ }^{2}$ \\ ${ }^{1}$ Mechanics of Materials, Sandia National Laboratories, 7011 East Avenue, Livermore, CA, U.S.A. \\ ${ }^{2}$ Numerical Analysis and Application, Sandia National Laboratories, PO Box 5800, Albuquerque, NM, U.S.A.
}

\begin{abstract}
SUMMARY
An adaptively stabilized finite element scheme is proposed for a strongly coupled hydro-mechanical problem in fluid-infiltrating porous solids at finite strain. We first present the derivation of the poromechanics model via mixture theory in large deformation. By exploiting assumed deformation gradient techniques, we develop a numerical procedure capable of simultaneously curing the multiple-locking phenomena related to shear failure, incompressibility imposed by pore fluid, and/or incompressible solid skeleton and produce solutions that satisfy the inf-sup condition. The template-based generic programming and automatic differentiation $(\mathrm{AD})$ techniques used to implement the stabilized model are also highlighted. Finally, numerical examples are given to show the versatility and efficiency of this model. Copyright (C) 2013 John Wiley \& Sons, Ltd.
\end{abstract}

Received 2 July 2012; Revised 9 November 2012; Accepted 10 November 2012

KEY WORDS: $\quad$ poromechanics; stabilized procedure; multiphase medium; bubble functions

\section{INTRODUCTION}

Poromechanics is a broad branch of mechanics aimed to predict how a porous solid behaves when infiltrated by pore fluid. It involves a wide spectrum of engineering applications, such as foundation design, reservoir management, hydraulic fracture, and bioengineering of tissue scaffolding. These applications motivate the development of various poromechanics models and their corresponding numerical schemes in the literature (cf. [1-7]).

Capturing the fully coupled physical process involving the solid and fluid phases is, nevertheless, not a trivial task. First, realistic physical simulations often require either an iterative sequential or monolithic solver to handle the multiple-field governing equations such that the Mandel-Cryer effect exhibited in porous media can be properly replicated $[5,8]$. If an iterative sequential scheme is used to couple the governing equations of the solid deformation and pore fluid flow, then both the solid and fluid mechanics solvers must undertake significant modification in $\mathrm{I} / \mathrm{O}$ design so that proper feedback and communication among solvers can be established [9]. Furthermore, sequential coupling may lead to instabilities when the load increment is small and the drained split strategy is used [10].

Alternatively, coupled poromechanics can be formulated and solved in a monolithic manner. Nevertheless, the monolithic approach is only valid if the fully coupled numerical scheme fulfills the inf-sup condition, a criterion to avoid numerical deficiencies due to an improper finite-dimensional space used in the finite element/difference discretization. There are three common choices used to obtain stable numerical solutions-by equally discretizing the solid skeleton displacement and

*Correspondence to: WaiChing Sun, WaiChing Sun, Mechanics of Materials, Sandia National Laboratories, Mail Stop 9912, 7011 East Avenue, Livermore, CA, U.S.A.

†E-mail:wsun@sandia.gov

Copyright (C) 2013 John Wiley \& Sons, Ltd. 
Darcy's velocity [7], by using inf-sup stable finite elements (e.g., Talyor-Hood, Raviart-Thomas finite elements) $[9,11-14]$, or by applying stabilization procedures to cure the otherwise unstable finite elements [7,15-18]. The displacement-Darcy-velocity coupling scheme is relatively easy to implement. However, computation time will be significantly increased because of the extra degrees of freedom added for the pore fluid velocity. Implementing inf-sup stable displacement-pressure finite element requires only one extra degree of freedom for the pore pressure. However, inf-sup stable finite elements require special meshing, data structure, and preprocessing and postprocessing tools to accommodate the need to have different basis functions for the displacement and pore pressure solutions. This difficulty is accompanied with a significant increase in computation cost, as higher-order mixed finite elements lead to larger systems of equations and require more integration points to perform numerical integration, as pointed out in [14].

An equal-order displacement-pore pressure mixed finite element formulation is computationally more efficient and does not require significant modification to data structures (except adding an additional degree of freedom to all nodes), and it is therefore more feasible for both code development and maintenance. The price for using the equal-order mixed finite element method, however, is that the mixed finite element formulation must be stabilized by either adding additional terms or introducing enhanced shape functions to eliminate the spurious mode encountered as a result of a failure to satisfy the inf-sup condition. In recent years, effort has been invested to develop stabilization procedures for poromechanics problem under the small strain assumption, for example, [7, 16-18]. However, there are numerous occasions in which porous solids experience significant deformation such that a finite strain formulation becomes essential. Examples include water-saturated soil near critical state [7] and hydrated biological tissue during normal physiological activities [19]. In those situations, a stabilized formulation for large deformation poromechanics inheriting the same numerical efficiency and flexibility of the small deformation counterpart is valuable.

Another important complication for the finite deformation poromechanics problem originates from the fact that the porous media may exhibit nearly incompressible constitutive responses not only at the undrained limit but also in (and not limited to) situations listed as follows:

- Both the fluid and solid constituents and the solid skeleton are nearly incompressible (e.g., marine clay [20] and soft tissue [19]).

- The deformation of the porous medium is bending dominated (e.g., bending of long bone [3] and gel beam [21]).

- The plastic flow of the elasto-plastic porous medium is isochoric (e.g., soil at critical state [22] or $J_{2}$ elasto-plastic materials).

In these cases, numerical difficulties may arise from the ill-conditioned tangent stiffness matrix and cause volumetric locking regardless of the drainage condition. To incorporate the kinematic constraint without volumetric locking, Levenston, Frank, and Grodzinsky [19] introduced an additional variable and included a Lagrange multiplier and a penalty Lagrangian functional into a Q1P0 finite element. In this case, the function space of the Lagrange multiplier must be chosen carefully to satisfy the inf-sup condition. Equal-order discretization on displacement, pore pressure, and the Lagrange multiplier is therefore not suitable.

To address the problem alluded to earlier, we propose a formulation that employs an assumed strain finite element technique in the definition for the deformation gradient to cure the volumetric locking exhibited in the poromechanics problems. Because both the usage of an assumed deformation gradient and equal-order basis functions for the displacement-pore pressure system may cause spurious modes from not satisfying the inf-sup condition (cf. [23]), we propose a simple polynomial pressure projection technique to stabilize the formulation. To the best knowledge of the authors, the numerical model presented in this paper is the first to propose stabilized equal-order poromechanics at finite strain, while simultaneously considering the volumetric locking effect and the compressibility of both solid and fluid constituents.

The rest of the paper is organized as follows. We first review the fundamentals of continuum mixture theory (Section 2). We next reformulate the variational form with equal-order discretization (Sections 3.1 and 3.2), apply the assumed deformation technique (Section 3.3), and discuss schemes for choosing stabilization parameters (Section 3.4). Furthermore, we also review how we apply a C++ 
template-based generic programming technique to significantly simplify the implementation procedure. In particular, we explain how the linearized Jacobian of the Galerkin form is obtained through $\mathrm{AD}$ tools, and therefore, we bypass the need to manually derive the geometric and material tangent stiffness (Section 4). Selected benchmark and engineering application problems are simulated via the stabilized formulations (Section 5). Finally, concluding remarks are given in Section 6.

As for notations and symbols, bold-faced letters denote tensors, the symbol '. ' denotes a single contraction of adjacent indices of two tensors (e.g., $\boldsymbol{a} \cdot \boldsymbol{b}=a_{i} b_{i}$ or $\boldsymbol{c} \cdot \boldsymbol{d}=c_{i j} d_{j k}$ ), the symbol ' $:$ ' denotes a double contraction of adjacent indices of tensor of rank two or higher (e.g., $\boldsymbol{C}: \boldsymbol{\epsilon}^{\boldsymbol{e}}=C_{i j k l} \epsilon_{k l}^{e}$ ), and the symbol ' $\otimes$ ' denotes a juxtaposition of two vectors (e.g., $\boldsymbol{a} \otimes \boldsymbol{b}=a_{i} b_{j}$ or two symmetric second-order tensors (e.g., $\left.(\boldsymbol{\alpha} \otimes \boldsymbol{\beta})=\alpha_{i j} \beta_{k l}\right)$. As for sign conventions, we consider the direction of the tensile stress and dilative pressure as positive.

\section{FUNDAMENTALS OF MIXTURE THEORY}

The large deformation theory of poromechanics has been studied in [5, 14, 19, 24, 25]. For completeness of this paper, we provide a brief overview and highlight the new contribution in this section. Here, we adopt a continuum approach to modeling the solid-fluid mixture that presumes that the various constituents of the porous media are well distributed throughout each configuration such that the mixture can be idealized as a multiphase macroscopic continuum via proper homogenization procedures $[2,26]$.

In addition, the following assumptions are made.

1. The isothermal condition holds.

2. Mass exchange between solid and fluid constituents does not occur.

3. Reynold's number of the flow in the pores is sufficiently low such that convection is negligible, and Darcy's law applies.

4. Inertial effects are negligible.

Notice that we consider both the solid and fluid constituents as compressible such that the formulation is valid for a wider range of materials, that is, porous media with Biot's coefficient less than one $\left(1-K / K_{s}<<1\right)$ and Biot's modulus being finite and measurable. Examples of those materials include limestone, sandstone, shale, and chalk.

\subsection{Kinematics}

In mixture theory, material points are occupied simultaneously by multiple constituents in a spatially averaged sense. Assuming the homogenization procedure is valid, multiple constituents may occupy a fraction of a representative elementary volume, and some of the physical quantities of the mixture, such as density and total traction, are then determined by spatially averaging those of the constituents. For instance, the total density of the mixture composed of a solid, and a fluid constituent reads

$$
\rho=\rho^{s}+\rho^{f}=\phi^{s} \rho_{s}+\phi^{f} \rho_{f}
$$

where $\rho_{\alpha}$ is the mass of the $\alpha$ constituent divided by the volume of the $\alpha$ constituent, whereas $\rho^{\alpha}$ is the intrinsic density of the $\alpha$ constituent, defined as the mass of the $\alpha$ constituent divided by the volume of the mixture.

Assume that the mappings between the material (Lagrangian) and the current (Eulerian) configurations for the solid and fluid constituents $\varphi^{\alpha}$ are sufficiently smooth, and then we have [27]

$$
\boldsymbol{x}=\varphi^{\alpha}\left(\boldsymbol{X}^{\boldsymbol{\alpha}}, t\right), \text { where } \alpha=s, f
$$

The mapping of the solid and fluid constituents is illustrated in Figure 1. Unlike the single-phase continua, multiphase continua are mixtures of constituents that are not bound to move under the same trajectories. Therefore, if a control mass of solid particles is defined in a solid phase material configuration, then the volume occupied by the same control mass of solid particles may contain pore fluid that does not belong to the solid phase material configuration. However, because the constitutive response of the solid skeleton must be modeled in a well-defined and measurable control mass at a continuum 


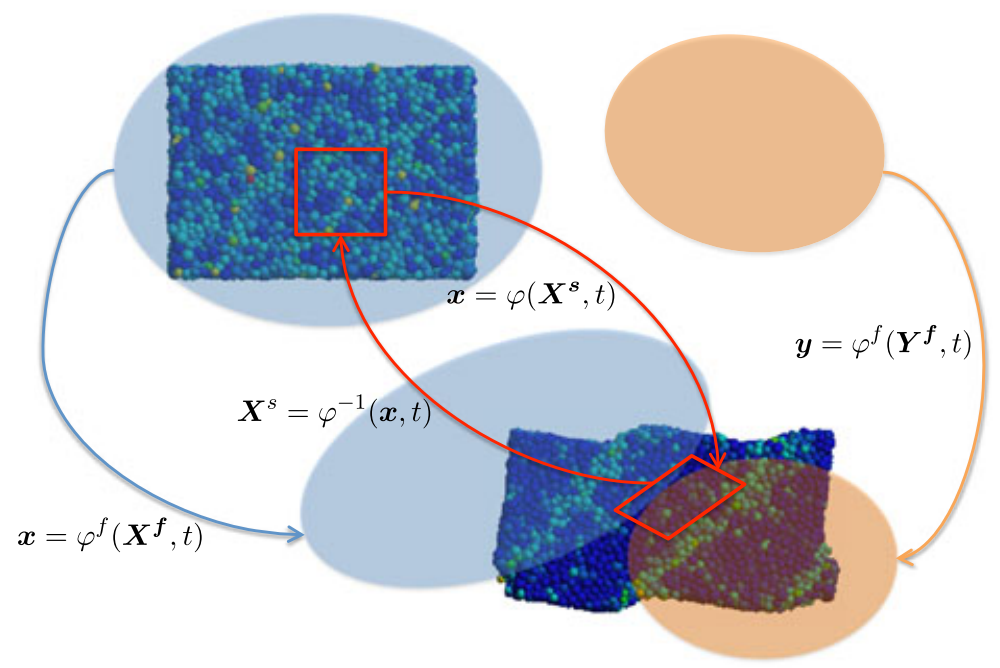

Figure 1. Trajectories of the solid and fluid constituents $\varphi^{s}=\varphi$ and $\varphi^{f}$. The motion $\varphi$ conserves all the mass of the solid constituent, whereas the fluid may enter or leave the body of the solid constituent. Figure reproduced from [28].

level [4], the finite strain poromechanics problem is formulated on the trajectory of the solid constituent only. The pore fluid motion is accounted indirectly by modeling the relative motion between the fluid flow and the solid skeleton through constitutive models such as Darcy's law. For brevity, we drop the designation of the solid phase $s$, such that $x=\varphi^{s}(\boldsymbol{X}, t)=\varphi(\boldsymbol{X}, t)$.

\subsection{Balance of linear momentum}

Provided that a macroscopic representation of the solid-fluid interaction in porous media is valid, the total Cauchy stress of a porous medium is the sum of the solid and fluid phase macroscopic Cauchy stress if the meniscus effect is neglected, that is,

$$
\sigma=\sigma^{s}+\sigma^{f}=\phi^{s} \sigma_{s}+\phi^{f} \sigma_{f}
$$

where $\sigma_{s}$ and $\sigma_{f}$ are the intrinsic partial stress defined in the volume of the solid grains $V^{s}$ and pores $V^{f}$, respectively. The total stress is the volume-averaged stress defined in the volume $V=V^{s}+V^{f}$, assuming that homogenization is valid. Because pore fluid does not provide any shear resistance, the fluid phase Cauchy stress is isotropic and holds the following relation with the macroscopic pore pressure $p^{f}$, that is,

$$
p^{f} \boldsymbol{I}=-\boldsymbol{\sigma}^{f}, \quad p^{f}=-\frac{1}{3} \operatorname{tr}\left(\boldsymbol{\sigma}^{f}\right), \quad p_{f}=-\frac{1}{3 \phi^{f}} \operatorname{tr}\left(\phi^{f} \sigma_{\boldsymbol{f}}\right)
$$

On the other hand, the Cauchy stress of the solid phase comes from the deformation of the solid skeleton (i.e., the effective stress $\sigma^{\prime}$ ) and the stress exerted on the skeleton due to the compression of the fluid (i.e., $K / K_{s} p^{f}$ ),

$$
\boldsymbol{\sigma}^{s}=\boldsymbol{\sigma}^{\prime}+\frac{K}{K_{s}} p^{f} \boldsymbol{I}
$$

This definition is from [29], which assumes that the nonuniform localization of stress at the grain scale, grain crushing, and damage are all insignificant to the skeleton (cf. [7] p. 8-11). By substituting (4) and (5) into (3), the total Cauchy stress now reads

$$
\sigma=\sigma^{\prime}-B p^{f} \boldsymbol{I}
$$


where $B$ is the Biot's coefficient defined as [29]

$$
B=1-\frac{K}{K_{s}}
$$

We will adopt the total first Piola-Kirchhoff stress $\boldsymbol{P}$ as the stress measure for the total Lagrangian formulation. The total first Piola-Kirchhoff stress is obtained through the Piola transformation [24,25]. For elastic porous media, the total first Piola-Kirchhoff stress can be determined from the deformation gradient $\boldsymbol{F}$ and the pore pressure $p^{f}$, that is,

$$
\boldsymbol{P}\left(\boldsymbol{F}, p^{f}\right)=\boldsymbol{P}^{\prime}(\boldsymbol{F})-J B p^{f} \boldsymbol{F}^{-T}
$$

where $J$ is the determinant of the deformation gradient $\boldsymbol{F}$. Notice that the effective first PiolaKirchhoff stress, $\boldsymbol{P}^{\prime}$, does not depend on the pore pressure $p^{f}$. On the other hand, if the constitutive response is path dependent, then we assume that the following relation holds:

$$
\boldsymbol{P}\left(\boldsymbol{F}, \mathbf{z}, p^{f}\right)=\boldsymbol{P}^{\prime}(\boldsymbol{F}, \mathbf{z})-J B p^{f} \boldsymbol{F}^{-T}
$$

where $\mathbf{z}$ is a set of internal variables. Notice that the effective first Piola-Kirchhoff stress defined in (9) is also decoupled from the pore fluid response. Hence, this assumption enables us to use any singlephase stress integration algorithm to obtain the effective first Piola-Kirchhoff stress. By applying standard mixture theory and neglecting the inertial force, the balance of linear momentum reads

$$
\begin{gathered}
\nabla^{\boldsymbol{X}} \cdot \boldsymbol{P}^{s}+J \rho^{s} \boldsymbol{G}+\boldsymbol{H}^{s}=\mathbf{0} \\
\nabla^{\boldsymbol{X}} \cdot \boldsymbol{P}^{\boldsymbol{f}}+J \rho^{f} \boldsymbol{G}+\boldsymbol{H}^{\boldsymbol{f}}=\mathbf{0}
\end{gathered}
$$

where $\rho^{\alpha}=\phi^{\alpha} \rho_{\alpha}$ is the intrinsic or apparent density of the $\alpha$ phase. $\boldsymbol{G}$ is the acceleration due to gravity. As pointed out in [27], $\boldsymbol{H}^{\boldsymbol{s}}$ and $\boldsymbol{H}^{\boldsymbol{f}}$ are the interactive body force per unit reference volume exerted on their corresponding phases due to drag, lift, virtual mass effect, history effects, and the relative spinning (Magnus effect) that balance out internally, that is, $\boldsymbol{H}^{\boldsymbol{s}}+\boldsymbol{H}^{\boldsymbol{f}}=\mathbf{0}$. As a result, combining (10) and (11) yields

$$
\nabla^{\boldsymbol{X}} \cdot \boldsymbol{P}+J\left(\rho^{s}+\rho^{f}\right) \boldsymbol{G}=\mathbf{0}
$$

or equivalently

$$
\nabla^{\boldsymbol{x}} \cdot \boldsymbol{\sigma}+\left(\rho^{s}+\rho^{f}\right) \boldsymbol{G}=\mathbf{0}
$$

where (12) and (13) are related by the Piola transformation $\nabla^{\boldsymbol{X}} \cdot \boldsymbol{P}=J \nabla^{\boldsymbol{x}} \cdot \boldsymbol{\sigma}$.

\subsection{Balance of mass}

Here, we derive a general balance of mass equation in which the compressibility of both the solid and pore fluid constituents are considered. Whereas this generalized derivation for compressible constituents has been recently considered in [13], our new contribution here is that the infinitesimal version of this formulation is fully consistent with the classical small strain balance law in $[1,7,29]$ and can also be consistently reduced to the finite strain formulation in [11] when the solid constituent becomes incompressible, and the finite strain formulation in $[2,25]$ when the porous media is fully saturated and composed of incompressible constituents.

Recall that we define the material time derivative on the basis of the motion of the solid skeleton. In the absence of mass exchange among all constituents, the balance of mass for the pore fluid constituent reads

$$
\frac{D \rho^{f}}{D t}=-\nabla^{\boldsymbol{X}} \cdot \boldsymbol{W}
$$


where $\boldsymbol{W}$ and $\boldsymbol{w}$ are the Lagrangian and Eulerian relative mass flow vectors defined as [5]

$$
\boldsymbol{w}=\phi^{f} \rho_{f}\left(\boldsymbol{v}^{f}-\boldsymbol{v}\right), \quad \boldsymbol{W}=J \boldsymbol{F}^{-1} \cdot \boldsymbol{w}
$$

Assuming isothermal conditions, the material time derivative of the pore fluid density can be partitioned through the following identity:

$$
\frac{D \rho^{f}}{D t}=\phi^{f} \frac{D \rho_{f}}{D t}+\rho_{f} \frac{D \phi^{f}}{D t}
$$

Notice that the material time derivatives can be further simplified as

$$
\frac{D \rho^{f}}{D t}=\rho^{f} \frac{D}{D t} \log \left(\frac{\rho_{f}}{\rho_{f 0}}\right)+\rho_{f} \frac{D \phi^{f}}{D t}
$$

where $\log \left(\rho_{f} / \rho_{f 0}\right)$ is the infinitesimal change of the pore fluid density. Assuming the first term of (17) reads

$$
\phi^{f}\left(\rho_{f} \frac{D}{D t} \log \left(\frac{\rho_{f}}{\rho_{f 0}}\right)\right)=\frac{\phi^{f} \rho_{f}}{K_{f}} \frac{D p^{f}}{D t}
$$

which is obtained by applying the barotropic assumption, as pointed out in [11],

$$
K_{f}=\rho_{f} \frac{d p^{f}}{d \rho_{f}}=\text { constant } \Rightarrow \frac{\rho_{f}}{\rho_{f 0}}=\exp \left(\frac{p^{f}}{K_{f}}\right)
$$

On the other hand, the second term takes into account the volumetric change of pore space for a fixed pore fluid density, which comes from the skeleton volumetric change and the volume changes caused by the compression or extension of the solid grains. Assuming that the change of porosity at an infinitesimal time is small, the change of porosity can be written as (cf. [30])

$$
\frac{D \phi^{f}}{D t}=\frac{D}{D t}\left(B \log J+\frac{B-\phi^{f}}{K_{s}} p^{f}\right)
$$

where $\log J=\log (\operatorname{det} \boldsymbol{F})=\operatorname{tr} \boldsymbol{\epsilon}$ and $\boldsymbol{\epsilon}$ is the Eulerian logarithm strain tensor. Applying the chain rule and working through the algebra, we obtain the following expression:

$$
\begin{aligned}
\frac{D \phi^{f}}{D t}= & \frac{K_{s}}{K_{s}+p^{f}}\left(\frac{D B}{D t}\left(\log J+\frac{p^{f}}{K_{s}}\right)\right) \\
& +\frac{K_{s}}{K_{s}+p^{f}}\left(\frac{B}{J} \frac{D J}{D t}+\frac{B-\phi^{f}}{K_{s}} \frac{D p^{f}}{D t}\right)
\end{aligned}
$$

For simplicity, we may consider only the case where $p^{f}<<K_{s}$ such that

$$
\frac{D \phi^{f}}{D t}=\frac{D B}{D t}\left(\log J+\frac{p^{f}}{K_{s}}\right)+\frac{B}{J} \frac{D J}{D t}+\frac{B-\phi^{f}}{K_{s}} \frac{D p^{f}}{D t}
$$

The material time derivative of the apparent pore fluid density reads

$$
\frac{D \rho^{f}}{D t}=\rho_{f}\left(\frac{D B}{D t}\left(\log J+\frac{p^{f}}{K_{s}}\right)+\frac{B}{J} \frac{D J}{D t}+\frac{1}{M} \frac{D p^{f}}{D t}\right)
$$


where $M$ is the Biot's modulus defined as [29]

$$
M=\frac{K_{s} K_{f}}{K_{f}\left(B-\phi^{f}\right)+K_{s} \phi^{f}}
$$

Finally, for completeness of presentation, we assume that the flow inside the porous media is sufficiently slow such that Darcy's law is valid. In this case, Darcy's flow can be used as constitutive model to relate the relative flow vector with the pore pressure. In the current configuration, the balance of linear momentum of the fluid phase may be written as

$$
-\nabla^{\boldsymbol{x}} p^{f}+\rho^{f} \boldsymbol{G}-\boldsymbol{k}^{-1} \cdot \boldsymbol{w}=\rho^{f} \boldsymbol{a}^{\boldsymbol{f}}
$$

where $\boldsymbol{k}$ is the permeability tensor divided by the viscosity and $\boldsymbol{a}^{f}$ is the acceleration of the fluid constituent. Rearranging (25), the Eulerian relative flow vector $\boldsymbol{w}$ reads

$$
\frac{1}{\rho_{f}} \boldsymbol{w}=\boldsymbol{k} \cdot\left[-\nabla^{\boldsymbol{x}} p^{f}+\rho_{f}\left(\boldsymbol{G}-\boldsymbol{a}^{f}\right)\right]
$$

Assume that the inertial force is negligible, $\boldsymbol{a}^{f}=\mathbf{0}$. By applying the Piola transformation of the relative flow vector and pulling back the permeability tensor, Darcy's law can be expressed in the Lagrangian configuration. The relative flow vector therefore reads

$$
\frac{1}{\rho_{f}} \boldsymbol{W}=\boldsymbol{K} \cdot\left(-\nabla^{\boldsymbol{X}} p^{f}+\rho_{f} \boldsymbol{F}^{\mathrm{T}} \cdot \boldsymbol{G}\right)
$$

where

$$
\boldsymbol{K}=J \boldsymbol{F}^{-1} \cdot \boldsymbol{k} \cdot \boldsymbol{F}^{-\mathrm{T}}
$$

Combining (14), (23), and (27), we obtain the strong form of the balance of mass equation,

$$
\frac{D B}{D t}\left(\log J+\frac{p^{f}}{K_{s}}\right)+\frac{B}{J} \frac{D J}{D t}+\frac{1}{M} \frac{D p^{f}}{D t}+\nabla^{\boldsymbol{X}} \cdot \boldsymbol{Q}=0
$$

where $\boldsymbol{Q}=\left(1 / \rho_{f}\right) \boldsymbol{W}$. Notice that if both constituents are incompressible, then $B=1$ and $1 / M=0$. By applying the Piola transform, (29) reduces to the form identical to that seen in [24],

$$
\nabla^{\boldsymbol{x}} \cdot \boldsymbol{v}+\nabla^{\boldsymbol{x}} \cdot \boldsymbol{q}=0
$$

where $\boldsymbol{q}=\left(1 / \rho_{f}\right) \boldsymbol{w}$.

\section{Remark 1}

Armero derived a quadratic potential to characterize the reversible response of the saturated pore space by assuming that the change of fluid content is small in [10]. In a special case where Biot's coefficient remains unchanged, (29) is identical to Equation (3.36) of [10].

\section{STABILIZED VARIATIONAL FORMULATION}

In this section, we consider the stabilized variational form required for the equal-order displacementpressure paired finite element model with assumed deformation gradient. We first define the standard weak form of the poromechanics problem on the basis of the balance law derived in Section 2. By applying a multiplicative split, we introduce the assumed deformation gradient suitable for the poromechanics problem. To prevent spurious modes due to the usage of equal-order interpolation and assumed deformation gradient, we introduce a stabilization mechanism into the weighted residual statement of the momentum and mass balance equations. A simple scheme for choosing the stabilization parameters is also presented. 


\subsection{Galerkin form}

Our objective is to derive a weighted residual statement suitable for a total Lagrangian formulation. We first specify the appropriate boundary and initial conditions. Following the standard line, we consider a domain $\mathcal{B}$ whose boundary $\partial \mathcal{B}$ is the direct sum of the Dirichlet and Von Neumann boundaries, that is,

$$
\begin{gathered}
\partial \mathcal{B}=\overline{\partial \mathcal{B}_{\boldsymbol{u}} \cup \partial \mathcal{B}_{\boldsymbol{t}}}=\overline{\partial \mathcal{B}_{p} \cup \partial \mathcal{B}_{q}} \\
\emptyset=\partial \mathcal{B}_{\boldsymbol{u}} \cap \partial \mathcal{B}_{\boldsymbol{t}}=\partial \mathcal{B}_{p} \cap \partial \mathcal{B}_{q}
\end{gathered}
$$

where $\partial \mathcal{B}_{\boldsymbol{u}}$ is the solid displacement boundary, $\partial \mathcal{B}_{\boldsymbol{t}}$ is the solid traction boundary, $\partial \mathcal{B}_{p}$ is the pore pressure boundary, and $\partial \mathcal{B}_{\boldsymbol{q}}$ is the pore fluid flux boundary. The boundary conditions are prescribed as

$$
\begin{gathered}
\boldsymbol{u}=\overline{\boldsymbol{u}} \text { on } \partial \mathcal{B}_{\boldsymbol{u}} \\
\boldsymbol{N} \cdot \boldsymbol{P}=\overline{\boldsymbol{t}} \text { on } \partial \mathcal{B}_{\boldsymbol{t}} \\
p^{f}=\bar{p} \text { on } \partial \mathcal{B}_{p} \\
-\boldsymbol{N} \cdot \boldsymbol{Q}=\bar{Q} \text { on } \partial \mathcal{B}_{\boldsymbol{Q}}
\end{gathered}
$$

In addition, we consider the trial space for the weak form that reads

$$
\begin{gathered}
V_{\boldsymbol{u}}=\left\{\boldsymbol{u}: \mathcal{B} \rightarrow \mathbb{R}^{3}\left|\boldsymbol{u} \in\left[H^{1}(\mathcal{B})\right]^{3}, \boldsymbol{u}\right|_{\partial B_{\boldsymbol{u}}}=\overline{\boldsymbol{u}}\right\} \\
V_{p}=\left\{p^{f}: \mathcal{B} \rightarrow \mathbb{R}\left|p^{f} \in H^{1}(\mathcal{B}), p^{f}\right|_{\partial_{B_{p}}}=\bar{p}\right\}
\end{gathered}
$$

where $H^{1}$ denotes the Sobolev space of degree one. The admissible variations of displacement $\eta$ and pore pressure $\psi$ therefore read

$$
\begin{gathered}
V_{\boldsymbol{\eta}}=\left\{\boldsymbol{\eta}: \mathcal{B} \rightarrow \mathbb{R}^{3}\left|\boldsymbol{\eta} \in\left[H^{1}(\mathcal{B})\right]^{3}, \boldsymbol{\eta}\right|_{\partial B_{\eta}}=\mathbf{0}\right\} \\
V_{\psi}=\left\{\psi: \mathcal{B} \rightarrow \mathbb{R}\left|\psi \in H^{1}(\mathcal{B}), \psi\right|_{\partial B_{p}}=0\right\}
\end{gathered}
$$

For brevity, the spatial argument $\boldsymbol{X} \in \mathcal{B}$ is not explicitly written. Moreover, the material time derivative of function $A$ is simply denoted as $\dot{A}$. The weighted residual statement of the balance of mass and linear momentum reads as follows: find $\boldsymbol{u} \in V_{\boldsymbol{u}}$ and $p^{f} \in V_{p}$ such that for all $\boldsymbol{\eta} \in V_{\boldsymbol{\eta} \mid}$ and $\psi \in V_{\psi}$,

$$
G\left(\boldsymbol{u}, p^{f}, \boldsymbol{\eta}\right)=H\left(\boldsymbol{u}, p^{f}, \psi\right)=0
$$

where $G: V_{\boldsymbol{u}} \times V_{p} \times V_{\eta} \rightarrow \mathbb{R}$ and $H: V_{\boldsymbol{u}} \times V_{p} \times V_{\psi} \rightarrow \mathbb{R}$ are defined as

$$
\begin{aligned}
G\left(\boldsymbol{u}, p^{f}, \boldsymbol{\eta}\right)= & \int_{\mathcal{B}} \nabla^{\boldsymbol{X}} \cdot \boldsymbol{\eta}: \boldsymbol{P}-J\left(\rho^{f}+\rho^{s}\right) \boldsymbol{\eta} \cdot \boldsymbol{g} \mathrm{dV} \\
& -\int_{\partial \mathcal{B}_{t}} \boldsymbol{\eta} \cdot \overline{\boldsymbol{t}} \mathrm{d} \Gamma \\
H\left(\boldsymbol{u}, p^{f}, \boldsymbol{\eta}\right)= & \int_{\mathcal{B}} \psi \dot{B}\left(\log J+\frac{p^{f}}{K_{s}}\right) \mathrm{dV} \\
& +\int_{\mathcal{B}} \psi \frac{B}{J} \dot{J}+\psi \frac{1}{M} \dot{p}^{f} \mathrm{dV} \\
& -\int_{\mathcal{B}} \nabla^{\boldsymbol{X}} \psi \cdot \boldsymbol{Q} \mathrm{dV} \\
& -\int_{\partial B_{Q}} \psi \overline{\boldsymbol{Q}} \mathrm{d} \Gamma
\end{aligned}
$$




\subsection{Time integration}

Because of the presence of the material time derivative of Biot's coefficient $B$, pore pressure $p^{f}$, and volumetric strain measure $J$, a time integration scheme is required. For the Biot's coefficient $B$ and pore pressure $p^{f}$, the solution is advanced via the backward Euler scheme where

$$
B_{n+1} \approx B_{n}+\dot{B}_{n+1} \Delta t, \quad p_{n+1}^{f} \approx p_{n}^{f}+\dot{p}_{n+1}^{f} \Delta t
$$

Notice that the backward Euler scheme alluded to earlier is not suitable for $J=\operatorname{det} \boldsymbol{F}, J \in \mathbb{R}^{+}$. For instance, a nonphysical negative $J_{n+1}$ may occur when $-\dot{J}_{n+1} \Delta t>J_{n}$ if the Backward Euler scheme is applied directly. The reason behind this error is that $J$ does not belong to the additive Abelian group in which addition is a group operation (cf.[31]). However, because $\log J \in \mathbb{R}$, we may take advantage of the following identity to integrate $J$ in time:

$$
J_{n+1}^{-1} \dot{J}_{n+1}=\frac{D}{D t}\left(\log J_{n+1}\right) \approx \frac{\log J_{n+1}-\log J_{n}}{\Delta t}
$$

Substituting (45) and (44) into weighted residual form (41), the time discretized weighted residual form reads

$$
\hat{G}\left(\boldsymbol{u}_{n+1}, p_{n+1}^{f}, \eta\right)=\hat{H}\left(\boldsymbol{u}_{n+1}, p_{n+1}^{f}, \psi\right)=0
$$

where

$$
\begin{aligned}
\hat{G}\left(\boldsymbol{u}_{n+1}, p_{n+1}^{f}, \boldsymbol{\eta}\right)= & \int_{\mathcal{B}} \nabla^{\boldsymbol{X}} \cdot \boldsymbol{\eta}: \boldsymbol{P}_{\boldsymbol{n}+\mathbf{1}} \mathrm{dV} \\
& -\int_{\mathcal{B}} J_{n+1}\left(\rho_{n+1}^{f}+\rho_{n+1}^{s}\right) \eta \cdot \boldsymbol{g} \mathrm{dV} \\
& -\int_{\partial \mathcal{B}_{t}} \boldsymbol{\eta} \cdot \overline{\boldsymbol{t}}_{\boldsymbol{n}+\mathbf{1}} \mathrm{d} \Gamma \\
\hat{H}\left(\boldsymbol{u}_{n+1}, p_{n+1}^{f}, \boldsymbol{\eta}\right)= & \int_{\mathcal{B}} \psi \frac{B_{n+1}-B_{n}}{\Delta t}\left(\log J_{n+1}+\frac{p_{n+1}^{f}}{K_{s}}\right) \mathrm{dV} \\
& +\int_{\mathcal{B}} \psi B_{n+1} \frac{\log J_{n+1}-\log J_{n}}{\Delta t} \mathrm{dV} \\
& +\int_{\mathcal{B}} \psi \frac{1}{M_{n+1}} \frac{p_{n+1}^{f}-p_{n}^{f}}{\Delta t} \mathrm{dV} \\
& -\int_{\mathcal{B}} \nabla^{\boldsymbol{X}} \psi \cdot \boldsymbol{Q}_{n+1} \mathrm{dV} \\
& -\int_{\partial B_{Q}} \psi \overline{\boldsymbol{Q}}_{n+1} \mathrm{~d} \Gamma
\end{aligned}
$$

\subsection{Enhanced deformation gradient for volumetric locking}

In this section, we derive an assumed deformation gradient for the case when the solid skeleton matrix becomes incompressible. A simple stabilization mechanism is provided.

The assumed deformation gradient method is often used to avoid the overconstraint associated with equal-order interpolations of the volumetric and isochoric parts of the deformation gradient $[23,32-34]$. The key to avoid this problem is to replace the interpolated volumetric deformation field $J=\operatorname{det} \boldsymbol{F}$ with a reduced order volumetric field $\bar{J}$ such that fewer volumetric constraints occur when incompressibility limit is approached. The resultant assumed deformation gradient is therefore composed of the modified volumetric deformation field and the original interpolated isochoric deformation gradient. 
Recall that the kinematic split of the deformation gradient $\boldsymbol{F}$ is formulated as

$$
\boldsymbol{F}=\boldsymbol{F}_{\text {vol }} \cdot \boldsymbol{F}_{\text {iso }}
$$

where

$$
\boldsymbol{F}_{\mathrm{vol}}=J^{1 / 3} \boldsymbol{I}, \quad \boldsymbol{F}_{\text {iso }}=J^{-1 / 3} \boldsymbol{F}
$$

An assumed strain formulation replaces the interpolated volumetric split $\boldsymbol{F}_{\text {vol }}=J^{1 / 3} \boldsymbol{I}$ with a modified definition $\overline{\boldsymbol{F}}_{\text {vol }}=\bar{J}^{1 / 3} \boldsymbol{I}$ such that

$$
\overline{\boldsymbol{F}}=\bar{J}^{1 / 3} \boldsymbol{F}_{\text {iso }}=\bar{J}^{1 / 3} J^{-1 / 3} \boldsymbol{F}
$$

However, the usage of an assumed deformation gradient may lead to numerical instabilities when the stiffness from the assumed deformation gradient is too low. As a result, Moran et al. (1990) [33] suggested replacing the assumed deformation gradient $\overline{\boldsymbol{F}}$ with a linear interpolation between the original and the assumed deformation gradient, that is,

$$
\widetilde{\boldsymbol{F}}=\alpha \boldsymbol{F}-(1-\alpha) \overline{\boldsymbol{F}}
$$

where $\alpha$ is a stabilization parameter in which $\alpha=0$ leads to the pure F-bar formulation and $\alpha=1$ leads to the standard formulation. The idea is to stiffen the element by increasing $\alpha$ whenever the numerical instability is encountered. Recent work by Mota et al. (2012), however, has demonstrated that linear interpolation may lead to significant error because the addition operation is not valid for the multiplicative group of which the deformation gradient belongs.

To provide the essential volumetric relaxation while maintaining stability, we introduce a simple combined/standard F-bar element by recourse to exponential/logarithmic mapping,

$$
\widetilde{\boldsymbol{F}}=\widetilde{J}^{1 / 3} J^{-1 / 3} \boldsymbol{F}
$$

where

$$
\widetilde{J}(X)=\exp \left(\frac{1-\alpha}{\mathrm{V}_{\mathcal{B}^{e}}} \int_{\mathcal{B}^{e}} \log J(X) \mathrm{dV}+\alpha \log J(X)\right)
$$

where $\alpha \in[0,1]$. The combined formulation may reduce to fully standard or F-bar formulation by adjusting $\alpha$ as in [33]. Furthermore, it can be easily shown that (53) is identical to the midpointassumed deformation gradient formulation in [32] if $\alpha=0$ and the volume averaging of $\log J(X)$ is computed via one-point quadrature at the centroid of the element.

Notice that this formulation does not require modification other than consistently replacing the conventional interpolated deformation gradient with the modified counterpart in (53).

\section{Remark 2}

The method to select $\alpha$ is at present unclear. Although the assumed deformation gradient may lead to spurious modes for certain single-phase solid mechanics problems as demonstrated in [35], nonzero $\alpha$ is not required in the solutions presented in the example section.

\subsection{Inf-sup condition and stabilization procedures}

Porous media with an incompressible fluid constituent may appear incompressible when pore fluid diffusion rate is much smaller than the solid deformation rate. This apparent incompressibility may cause spatial spurious pressure modes if the equal-order mixed, fully coupled standard Galerkin finite element formulation is used without a stabilization procedure. As pointed out in [18], this spurious mode is most severe if the time step is fine and/or the permeability is low.

Similar spurious pressure modes have also been observed in semidiscrete transient single-field, unsteady porous flow problems at small time steps or when hydraulic conductivity is significantly lower than the hydraulic storage (cf. [36]). This problem is a manifestation of the degradation of stability in the $H^{1}$ sense because maintaining $L_{2}$ stability of the finite element solution does not help 
controlling the spatial derivatives. Unlike the first case, this spurious oscillation occurs in coupled poromechanics problems even if an inf-sup stable displacement-pore-pressure finite element space (e.g., Taylor-Hood) is used.

Truty and Zimmermann [17] adopted the stabilization procedure for the stationary Stokes equations proposed in [37] for small strain poromechanics (referred to as the fluid pressure Laplacian method therein). This approach uses the element-wise integrals of the pressure gradient rate to filter out the spurious modes. Preisig and Prevost analyzed the performance of this method and introduced a lower bound to the stabilization parameter based on a one-dimensional (1D) analysis [16].

On the other hand, another pressure projection technique originally developed for the stationary Stokes' equation in [38] has been adopted to small strain poromechanics problems in [18]. In this work, the interpolated rate of change of pore pressure is projected onto an element-wise constant function to derive the stabilization term. The stabilization parameter is recommended to be $\tau / 2 G$, where $\tau$ is a free tuning parameter and $G$ is the shear modulus. Numerical examples demonstrate that $\tau=1$ is a good starting point for most problems, although some tuning of $\tau$ may still be required.

Our new contributions here are twofold. First, we point out that the stabilization methods in [17] and [18] can both be derived from the weak inf-sup condition. Although a similar conclusion has been reached for Darcy's and Stokes' problems in [39], this relation has not been pointed out in the poromechanics literature yet. Hence, the fluid pressure Laplacian method and the pressure projection method are sometimes regarded as different methods, for instance, in [16].

Second, we provide a new derivation to adaptively estimate the stabilization parameters for both methods accordingly. The resultant stabilization formulation adaptively adjusts the stabilization and requires no additional input from users to tune the stabilization parameter in most situations.

3.4.1. Weak inf-sup condition and stabilization procedures. Consider the update of a finite element solution in a sufficiently fine time step such that spurious modes occur in the standard Galerkin approximation. At such a small time scale, we assume that the deformation is infinitesimal such that derivatives in material and current configuration are approximately the same. Hence, a stable and accurate approximation of the displacement-pressure mixed finite element problem requires the pair of $V_{p}^{h}$ and $V_{\boldsymbol{u}}{ }^{h}$ satisfying the discrete inf-sup condition, that is,

$$
\sup _{\boldsymbol{u}^{h} \in V_{\boldsymbol{u}} h} \frac{\int_{B} p^{h} \nabla^{\boldsymbol{X}} \cdot \boldsymbol{u}^{h} \mathrm{dV}}{\left\|\boldsymbol{u}^{h}\right\|_{1}} \geqslant \gamma\left\|p^{h}\right\|_{0}, \forall p^{h} \in V_{p}^{h}
$$

where $\gamma$ is a positive constant independent of the mesh size. Although equal-order pairs $V_{p}^{h}$ and $V_{\boldsymbol{u}}{ }^{h}$ do not satisfy (55), the analysis performed by Verfürth [40] can be applied to the poromechanics problem to show that a weaker inf-sup condition is satisfied for a continuous displacement and pore pressure function.

$$
\sup _{\boldsymbol{u}_{h} \in V_{\boldsymbol{u}} h} \frac{\int_{B} p_{h} \nabla^{\boldsymbol{X}} \cdot \boldsymbol{u}_{h} \mathrm{dV}}{\left\|\boldsymbol{u}_{h}\right\|_{1}}+\gamma_{2}\left(\sum_{K \in \Omega} h_{K}^{2}\left\|\nabla^{\boldsymbol{X}} p^{h}\right\|_{0, K}\right)^{\frac{1}{2}} \geqslant \gamma_{1}\left\|p^{h}\right\|_{0}, \forall p^{h} \in V_{p}^{h}
$$

where $\gamma_{1}$ and $\gamma_{2}$ are both positive constants independent of the mesh size and $K$ is the finite element with element size $h_{K}$. A simple remedy to restore stability is directly adding the second term in (56) such that the inf-sup deficiency is counterbalanced. This method leads to an additional stabilization term in the mass balance equation, which reads

$$
R^{\mathrm{stab}}\left(\psi, p_{\tau}^{f}\right)=\sum_{K \in \Omega} h_{K} \int_{K} \nabla^{\boldsymbol{X}} \psi \cdot \beta \boldsymbol{K} \nabla^{\boldsymbol{X}} p_{\tau}^{f} \mathrm{dV}
$$

where $\boldsymbol{K}$ is defined in (28) and $\beta$ has a dimension of 1/length. $p_{\tau}^{f} \in V_{p}$ is the pore pressure at time $\tau$. This stabilization term leads to the fluid pressure Laplacian scheme in [17]. Alternatively, one may 
apply the standard inverse inequality onto the second term in (56), which leads to

$$
\begin{aligned}
\sum_{K \in \Omega} h_{K}^{2}\left\|\nabla^{\boldsymbol{X}} p^{h}\right\|_{0, K} & =\sum_{K \in \Omega} C\left\|\nabla^{\boldsymbol{X}}\left(p^{h}-\Pi p^{h}\right)\right\|_{0, K} \\
& \leqslant \sum_{K \in \Omega} C\left\|p^{h}-\Pi p^{h}\right\|_{0, K}
\end{aligned}
$$

where $C$ is a positive constant independent of the element mesh size, $\Pi$ is an $L_{2}$ projection operator, and $\Pi p^{h}$ is the $L_{2}$ projection onto the piecewise constant. Applying (58) on (56) leads to

$$
\begin{aligned}
\sup _{\boldsymbol{u}^{h} \in V_{\boldsymbol{u}}} \frac{\int_{B} p^{h} \nabla^{\boldsymbol{X}} \cdot \boldsymbol{u}^{h} \mathrm{dV}}{\left\|\boldsymbol{u}^{h}\right\|_{1}}+\sum_{K \in \Omega} \gamma_{3}\left\|p^{h}-\Pi p^{h}\right\|_{0, K} \geqslant & \sup _{\boldsymbol{u}^{h} \in V_{\boldsymbol{u}}} \frac{\int_{B} p^{h} \nabla^{\boldsymbol{X}} \cdot \boldsymbol{u}^{h} \mathrm{dV}}{\left\|\boldsymbol{u}^{h}\right\|_{1}} \\
& +\gamma_{4}\left(\sum_{K \in \Omega} h_{K}^{2}\left\|\nabla^{\boldsymbol{X}} p^{h}\right\|_{0, K}\right)^{\frac{1}{2}} \\
& \geqslant \gamma_{5}\left\|p^{h}\right\|_{0}, \forall p^{h} \in V_{p}^{h}
\end{aligned}
$$

where $\gamma_{3}, \gamma_{4}$, and $\gamma_{5}$ are positive constants independent of the element mesh size. As shown in (59), the inf-sup deficiency is bounded by the norm of the difference between the pore pressure field and its $L_{2}$ projection. The corresponding stabilization term is therefore to add this difference back to the continuity equation, which leads to the pressure projection stabilization scheme in [18], that is,

$$
R^{\mathrm{stab}}\left(\psi, p_{\tau}^{f}\right)=\sum_{K \in \Omega} \int_{K}(\psi-\Pi \psi) \frac{\gamma}{M^{\prime}}\left(p_{\tau}^{f}-\Pi p_{\tau}^{f}\right) \mathrm{dV}
$$

where $M^{\prime}$ has the dimension of the Biot's modulus $M$. The stabilization parameter $\gamma$ is therefore dimensionless. We will provide physical interpretation of $M^{\prime}$ in Section 3.4.2. Notice that $R^{\text {stab }}$ defined in (57) and (60) can be both considered as the virtual volumetric deformation corresponding to the penalty energy that regularizes the gradient of pore pressure by applying the first Gateaux variation of the following penalty energy functional at time step $\tau$, as shown in (61) and (62).

$$
\begin{gathered}
W^{\text {pen }}\left(p_{\tau}^{f}\right)=\frac{1}{2} \sum_{K \in \Omega} \int_{K}\left(p_{\tau}^{f}-\Pi p_{\tau}^{f}\right) \frac{\gamma}{M^{\prime}}\left(p_{\tau}^{f}-\Pi p_{\tau}^{f}\right) \mathrm{dV} \\
W^{\text {pen }}\left(p_{\tau}^{f}\right)=\frac{1}{2} \sum_{K \in \Omega} h_{K} \int_{K} \nabla^{\boldsymbol{X}} p_{\tau}^{f} \cdot \beta \boldsymbol{K} \nabla^{\boldsymbol{X}} p_{\tau}^{f} \mathrm{dV}
\end{gathered}
$$

Similar penalty energy-based stabilization approaches have been discussed in the content of meshless, nodal averaging and nodal deformation gradient methods in [35,41,42].

Finally, applying the stabilized formulation in temporal discrete variational equation (46) yields

$$
\hat{G}\left(\boldsymbol{u}_{n+1}, p_{n+1}^{f}, \boldsymbol{\eta}\right)=\hat{H}^{\prime}\left(\boldsymbol{u}_{n+1}, p_{n+1}^{f}, \psi\right)=0
$$

where

$$
\begin{aligned}
\hat{H}^{\prime}\left(\boldsymbol{u}_{n+1}, p_{n+1}^{f}, \psi\right)= & \hat{H}\left(\boldsymbol{u}_{n+1}, p_{n+1}^{f}, \psi\right) \\
& +R^{\text {stab }}\left(\psi, p_{n+1}^{f}-p_{n}^{f}\right)
\end{aligned}
$$

In summary, the aforementioned derivation proves the existence of a stabilization parameter, which ensures that the solution satisfies the weaker inf-sup condition, and establishes the relation between the stabilization term and penalty energy functionals. The estimation of the stabilization parameters for specific methods is discussed in the next section. 
3.4.2. Stabilization parameter estimation. Although (59) proves that spurious modes can be eliminated for a sufficiently large stabilization parameter, it does not give information on how large the stabilization parameter should be. Although one may assign a very large value to stabilize the solution, doing so may overdiffuse the solution and lead to nonphysical results [18].

Because it is difficult to directly determine, a priori, the optimal value of the stabilization parameter for multidimensional large deformation poromechanics problems, we estimate the stabilization parameter on the basis of a simplified 1D diffusion problem. As [36] points out, this represents a uniform $d$-dimensional mesh aligned with the direction of the growth and decay of the solution.

Our starting point of this analysis is the linearized perturbation equation from Rice [43], where an infinitesimal perturbation of pore pressure $\hat{p}$ satisfies the linearization of the 1D poromechanics governing equation, written as

$$
c \frac{\partial^{2} \hat{p}}{\partial x^{2}}=\frac{\partial \hat{p}}{\partial t}
$$

where $c$ is the diffusivity. If the elastic response of the solid skeleton and the permeability are both isotropic and the plastic response is of the Drucker-Prager type, then the diffusivity $c$ can be expressed as

$$
c=\frac{k}{\mu} \frac{M^{\prime} H}{H+v \beta M^{\prime}}, \quad M^{\prime}=\frac{M(K+4 G / 3)}{K+4 G / 3+B^{2} M}
$$

where $H, v$, and $\beta$ are the hardening modulus, dilatancy factor, and frictional parameter determined from the constitutive model. Notice that $M^{\prime}$ also appears in (60) and (61).

For brevity, the derivation of $(65)$ is not repeated here. Interested readers please refer to $[30,43,44]$ for details.

Equations taking the form of (65) can be discretized in time and that leads to a modified Helmholtz operator in space [36], which reads

$$
\hat{p}_{n+1}+\vartheta c \Delta t \frac{\partial^{2}}{\partial x^{2}} \hat{p}_{n+1}=\hat{p}_{n}-(1-\vartheta) c \Delta t \frac{\partial^{2}}{\partial x^{2}} \hat{p}_{n}
$$

where $\vartheta \in[0,1]$ is the scalar parameter of the generalized trapezoid rule. Without introducing any boundary conditions, (67) has an exact solution that reads [36]

$$
\hat{p}(x)=\exp ( \pm x / \sqrt{\vartheta c \Delta t})
$$

Notice that the finite element approximate growth and decay rate of the solution can be determined by assuming that $x_{A}=A h$ such that

$$
\hat{p}_{A}=\exp \left( \pm\left(h /(\sqrt{\vartheta c \Delta t})^{h}\right)^{A}\right.
$$

where $\sqrt{\vartheta c \Delta t}^{h}$ is the approximate growth/decay term in the finite element solution. Obviously, the finite element solution may exhibit spurious oscillations if $\sqrt{\vartheta c \Delta t}^{h}$ is complex valued, as pointed out in [36]. To determine the range of the stabilization parameters that prevent $\sqrt{\vartheta c \Delta t}^{h}$ being complex valued, we consider a simple case in which piecewise-linear finite elements are of uniform size $h$. As a result, we may construct a three-node stencil via the standard Galerkin method, that is,

$$
-\hat{p}_{A-1}+2 \hat{p}_{A}-\hat{p}_{A-1}+\frac{h^{2}}{6 \vartheta c \Delta t}\left(\hat{p}_{A-1}+4 \hat{p}_{A}+\hat{p}_{A+1}\right)=0
$$

Next, we add the stabilization terms defined in (57) and (60) into (67). By assembling the effective stiffness matrices of the $L_{2}$ projection stabilization term, we obtain

$$
-\hat{p}_{A-1}+2 \hat{p}_{A}-\hat{p}_{A-1}+\frac{h^{2}}{12 \vartheta c \Delta t}\left[(2-\gamma) \hat{p}_{A-1}+(8+2 \gamma) \hat{p}_{A}+(2-\gamma) \hat{p}_{A+1}\right]=0
$$


where $\gamma$ is the stabilization parameter for the pressure projection term. On the other hand, the three-node stencil stabilized by the fluid pressure Laplacian method reads

$$
\left(1+\beta \frac{h k}{\vartheta \Delta t \mu}\right)\left(-\hat{p}_{A-1}+2 \hat{p}_{A}-\hat{p}_{A-1}\right)+\frac{h^{2}}{6 \vartheta c \Delta t}\left(\hat{p}_{A-1}+4 \hat{p}_{A}+\hat{p}_{A+1}\right)=0
$$

where $\beta$ is the stabilization parameter for the gradient stabilization term. By comparing (71) and (72), one may show that the $L_{2}$ projection stabilization and fluid pressure Laplacian stabilization are identical to each other in the $1 \mathrm{D}$ case if the following holds:

$$
\gamma=\left(12 \frac{c k}{\mu h}\right) \beta
$$

Hence, once the bound for the stability parameter $\gamma$ is defined, the bound of $\beta$ is also known via (73). To compute the stability bound for the $L_{2}$ projection stabilization, we first apply (69) into (71), which leads to

$$
\cosh \frac{h}{(\sqrt{\vartheta c \Delta t})^{h}}=\frac{\left(1+h^{2} / \vartheta c \Delta t\right)(4+\gamma) / 6}{\left(1-h^{2} / \vartheta c \Delta t\right)(2-\gamma) / 12}
$$

The approximate growth/decay rate does not contain an imaginary part if the hyperbolic cosine term is positive. Provided that $\gamma$ and $\beta$ are both positive, the stabilization parameter that eliminates spurious oscillation can be determined from the denominator in the right-hand side of (74),

$$
\gamma>2-12 \frac{\vartheta c \Delta t}{h^{2}}>0
$$

which is equivalent to the following relation for the 1D case:

$$
\beta>\frac{\mu}{k}\left(\frac{c h}{6}-\frac{\vartheta k \Delta t}{\mu h}\right)>0
$$

In our implementation, we employ the $L_{2}$ projection scheme and define $\gamma$ as

$$
\gamma=\left(2-6 \frac{\vartheta c \Delta t}{h^{2}}\right)\left(\frac{1}{2}+\frac{1}{2} \tanh \left(2-12 \frac{\vartheta c \Delta t}{h^{2}}\right)\right)
$$

Notice that the term $1 / 2+\tanh \left(2-12 \frac{\vartheta c \Delta t}{h^{2}}\right) / 2$ approaches zero if diffusivity is high and equals to 1 if diffusivity is low. This treatment is to limit overdiffusion caused by usage of the stabilization term as mentioned in [18].

\section{Remark 3}

It is evident that the estimation of the stabilization parameter is based on a $1 \mathrm{D}$ problem and thus as useful as it is, cannot be relied upon as a definitive analytical solution for the optimal value of the stabilization parameter. Nevertheless, in engineering practice, it may serve as an useful guideline for typical problems. In the numerical examples shown in Section 5, the estimated stabilization parameter is able to eliminate spurious oscillations and converges to analytical solutions without introducing significant overdiffusion.

\section{Remark 4}

Notice that the aforementioned formulation can be reduced to the classical 1D lumped mass case if $\gamma=2$ and $\beta=h /(6 \vartheta c \Delta t)$. The latter relation has been pointed out in [16]. The stabilization parameter suggested in [18] is equivalent to $\gamma=2 M^{\prime} / G$ in our formulation. This is a more conservative choice than the $\gamma$ defined in (77) if $1 / 2 G$ is larger than $2 / M^{\prime}$.

\section{Remark 5}

Rice's analysis in [43] has shown that dilatant hardening is unstable when $H$ is negative. This unstable response prevails in both analytical and numerical responses because the growth/decay rates of the numerical and analytical solutions are both complex valued. 


\section{Remark 6}

For multidimensional problems, one may use the definition in [45] to define the element length, that is,

$$
h(\boldsymbol{X})=2\left(\sum_{a}\left|\frac{\nabla^{\boldsymbol{X}} p^{f}(\boldsymbol{X})}{\left\|\nabla^{\boldsymbol{X}} p^{f}(\boldsymbol{X})\right\|} \cdot \nabla^{\boldsymbol{X}} N_{a}(\boldsymbol{X})\right|\right)^{-1}
$$

where $h(\boldsymbol{x})$ is not a constant within an element, but rather a continuous field that measures the element length in the direction of the pore pressure gradient. This definition, however, is not suitable for problems where pore pressure varies within the boundary layer but remains zero elsewhere. For those cases, we define the element length as

$$
h(\boldsymbol{X})=2\left(\sum_{a}\left|\boldsymbol{N} \cdot \nabla^{\boldsymbol{X}} N_{a}(\boldsymbol{X})\right|\right)^{-1}
$$

where $N=(1 / \sqrt{3})\left(\boldsymbol{e}_{1}+\boldsymbol{e}_{2}+\boldsymbol{e}_{3}\right)$ is a unit vector.

\section{IMPLEMENTATION}

Implementation of the poromechanics formulation presented earlier is carried out within a highly abstracted $\mathrm{C}++$ framework employing template-based generic programming practices. The motivation and advantages of such an environment are presented in this section and include access to transformational tools, graph-based assembly, simplified analytic linearization, and a natural treatment of strongly coupled systems. The section summarizes the framework described in [46] and [47].

Demands on multiphysics analyses, including poromechanics, uncertainty quantification, optimization, and sensitivity analysis, require additional embedded computational capabilities. These embedded tools have been implemented using templates and operator overloading in a series of packages within the Trilinos framework [48]. These packages have been employed in an abstracted finite element code, Albany. Using template-based generic programming concepts, fundamental operations are abstracted, and the resulting interfaces are used to compute additional quantities. To achieve this, fundamental physical quantities necessary in the computation are implemented independently as evaluators, with well-defined and managed dependencies. Examples include the deformation gradient and the permeability. A collection of evaluators can be connected to compute the residual, for example. This collection of evaluators is naturally expressed as a directed graph, where the evaluator dependencies can be uniquely sorted and determined at run time and visualized for debugging purposes. Furthermore, each evaluator is templated on type, where nonstandard types employ operator overloading techniques to permit computation of the advanced concepts mentioned earlier. More information about the particular Trilinos package employed to handle the dependency management and graph creation can be found in [49] and [50].

Once the evaluators have been sorted, the computation of the residual consists of a templated seedcompute-extract phase. The seed phase gathers data from global data structures to be used in local computations, whereas the extract phase takes the computed local fields and scatters them back to the global data structures. Template specialization is used during the seed and compute phases to properly initialize embedded types (seed) and transform embedded computed information (extract). The compute phase is written on the generic template type. A schematic of this approach can be seen in Figure 2.

An important consequence of the templated element-based assembly is clear in the context of solving nonlinear coupled systems of PDEs. Deriving and implementing the nonlinear Jacobian, including all of the coupling terms, are a time-consuming and error-prone process. Nevertheless, an analytic Jacobian is highly desirable as it vastly reduces the cost of a simulation in terms of the number of iterations. The employment of $\mathrm{AD}$, which is one of the embedded advanced types alluded to earlier, facilitates the usage of an analytic Jacobian for the coupled system without the need to derive and implement the Jacobian sensitivities each time a model is changed. Specifically, during the computation of the Jacobian, the seed phase initializes the sensitivities with respect to the independent variables, namely the degrees of freedom in the problem. The key is that computing the Jacobian terms 

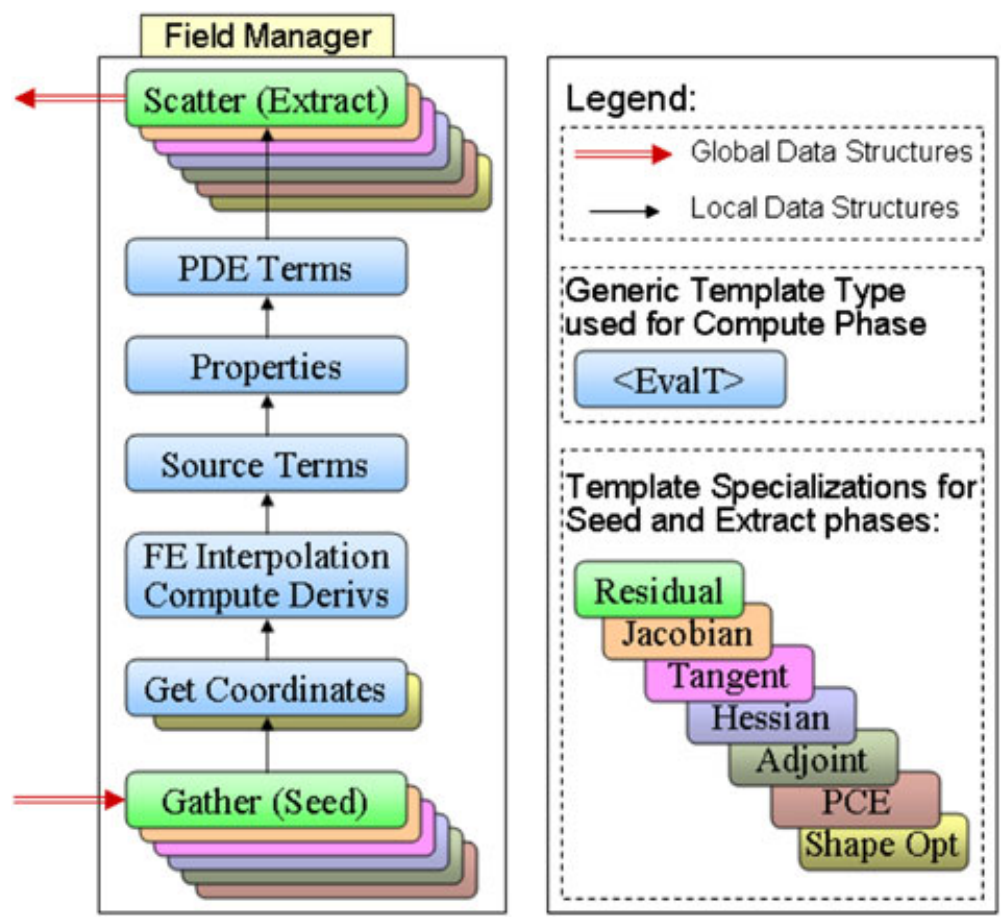

Figure 2. The template-based generic programming model as illustrated by a seed-compute-extract algorithm.

is now simply the result of computing the residual by using the $\mathrm{AD}$ type. The following extract phase scatters the Jacobian terms into the global system matrix, and the resulting system inherits the analytic Jacobian. It should be clear that a change in physics now only requires a modification to the general templated compute phase, written in terms of the residual, and the accompanying change in the Jacobian is simply a result of changing the residual. More information about AD as implemented in Trilinos can be found at [51].

Managed dependencies and graph-based assembly working in conjunction with embedded AD creates a powerful framework in which to explore strongly coupled physical systems. The poromechanics formulation discussed in the present work leverages this framework to achieve fully coupled, monolithic systems with analytic Jacobians. The next section will provide some numerical examples taking full advantage of these capabilities.

\section{Remark 7}

It should be noticed that implicit monolithic poromechanics problems often lead to ill-conditioned Jacobians that are difficult to solve in an efficient and scalable manner. Although direct solvers may handle small-sized problems, their performance may be severely hindered by memory bottlenecks. One possible remedy is to use iterative solver with block preconditioner [52].

\section{NUMERICAL EXAMPLES}

We provide numerical examples to verify the correctness of the implementation, demonstrate the stability of the numerical solutions and the improved element performance in bending dominated problems, and highlight the difference between the small and large deformation formulations of the poromechanics models. Results obtained from large deformation simulations are compared with small strain finite element simulations and analytical solutions, whenever possible. The topic of the 
numerical examples is selected from various engineering disciplines to demonstrate the general applicability of the formulation, as listed in the following:

- 1D cartilage problem.

- 3D elastic bending problem with incompressible soft tissue.

- 2D plane strain elasto-plastic footing problem.

- 3D circular footing.

Both small and finite deformation versions of the equal-order mixed finite element formulations are implemented. All stabilized numerical solutions are obtained from the $L_{2}$ projection scheme.

\subsection{Stress relaxation hydrated articular cartilage}

Articular cartilage is one of the major connective tissues that provides a low-friction bearing surface to distribute stress across joints and also functions as shock absorber. It contains no blood vessels, lymph channels, and nerves but contains $60 \%-85 \%$ of water in weight [3]. As a result, the mechanical response of articular cartilage significantly depends on the diffusion of water inside the cell matrix. Under the assumption of small strain and constitutive linearity, Haider and Guilak [53] formulated the poroelasticity problem in Laplace transform domains to simulate the stress relaxation in a cartilage layer under uniaxial confined compression. For verification purposes, we extend the classical small strain boundary value problem in $[3,53]$ to the finite deformation regime and compare the results obtained from a stabilized finite element simulation against the analytical solution.

For the sake of completeness, we briefly outline the boundary value problem of the uniaxial confined compression of cartilage layer reproduced from [53]. First, a fully saturated stress free articular cartilage specimen is placed in a cylindrical hydrated chamber such that no pore fluid exchange occurs at the bottom and the side boundaries. As a result, the pore fluid flux is zero there. A ramp-and-hold compressive displacement is applied on the top surface where water is free to flow in and out the articular layer, that is,

$$
u_{z}(x, y, H, t)=\left\{\begin{array}{l}
-\gamma t \text { if } 0 \geqslant t \geqslant t_{o} \\
-\gamma t_{o} \text { if } t>t_{0}
\end{array}\right\}
$$

where $h=30$ is the height of the cylinder and the radius of the cylinder is 30 . The analytical solution of this problem is available if both constituents are assumed to be incompressible and the permeability tensor is isotropic, in which the traction on the top surface reads

$$
\begin{aligned}
\left.t_{3}(t)\right|_{z=h}= & \frac{\gamma H_{A}}{h}\left[t H\left(t_{o}-t\right)+t_{o} H\left(t-t_{o}\right]\right. \\
& +\frac{2 \gamma h}{\kappa} \sum_{n=1}^{\infty} \frac{1}{n^{2} \pi^{2}}\left[H\left(t_{o}-t\right)\left(1-e^{-a_{n} t}\right)\right. \\
& \left.+H\left(t-t_{o}\right)\left(e^{-a_{n}\left(t-t_{o}\right)}-e^{-a_{n} t}\right)\right]
\end{aligned}
$$

where $H_{A}=\lambda+2 G$ is the sum of the Lamé's first parameter and two times the shear modulus, $a_{n}=n^{2} \pi^{2} \kappa H_{A} / h^{2}$, and $\kappa=k / v$ is the intrinsic permeability $k$ divided by the viscosity. Because of the axisymmetry, only a quarter of the domain is modeled. This domain is discretized by 1036 trilinear hexahedral finite elements via a radial mesh algorithm from a software called CUBIT [54] developed by the Sandia National Laboratories. The material parameters are listed in Table I. A compressible neo-Hookean material model is implemented as an extension of the isotropic linear Hooke's law. The first Piola-Kirchhoff stress tensor is obtained via following Helmholtz free energy [55],

$$
\begin{gathered}
\Psi^{s}(\boldsymbol{C})=\frac{G}{2}\left(J^{-\frac{2}{3}} I_{1}-3-2 \log J\right)+\frac{K}{2}(J-1)^{2} \\
\boldsymbol{P}=2 \boldsymbol{F} \frac{\partial \Psi^{s}(\boldsymbol{C})}{\partial \boldsymbol{C}}
\end{gathered}
$$


Table I. Material parameter used in confined compression stress relaxation problem.

\begin{tabular}{ll}
\hline Parameter & \multicolumn{1}{c}{ Value } \\
\hline Initial porosity & 0.5 \\
Solid grain bulk modulus & $50 \times 10^{12}$ \\
Pore fluid bulk modulus & $20 \times 10^{12}$ \\
Initial intrinsic permeability & $8.3333 \times 10^{-5}$ \\
Viscosity & 1.0 \\
Poisson's ratio & 0.25 \\
Young's modulus & $10^{5}$ \\
\hline
\end{tabular}

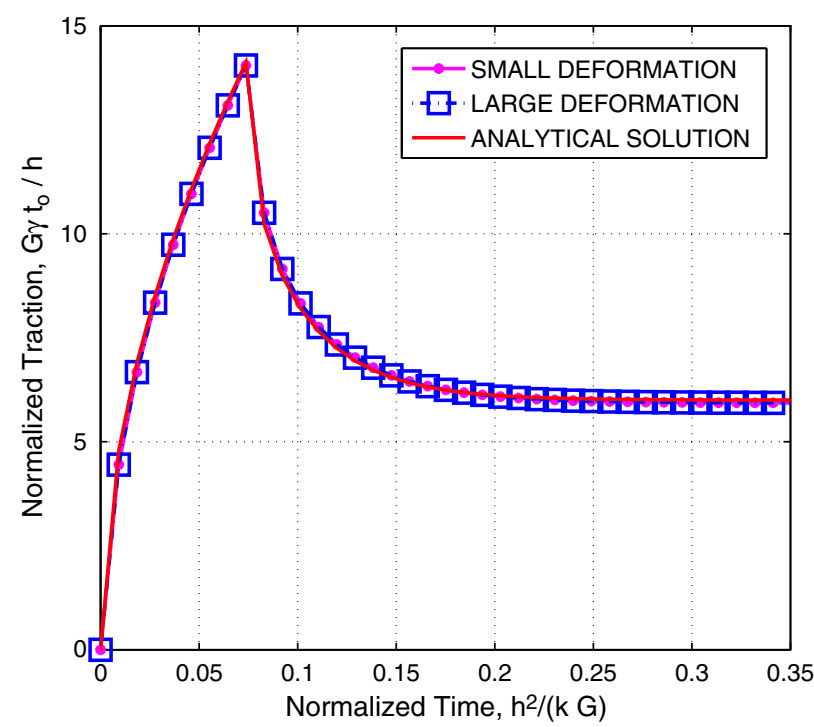

Figure 3. Dimensionless traction response obtained via stabilized finite element method and analytical solution at the top of the fully saturated specimen $\left(\gamma / L_{o}=0.005\right.$ and $\left.t_{o}=2.0\right)$.

where $I_{1}$ is the first invariant of $\boldsymbol{C}$, the right Cauchy-Green deformation tensor. Because the Poission's ratio is not close to the incompressible limit, the enhanced deformation gradient option is turned off. We conduct both small and large strain finite element simulations and compare the results against the analytical solution with various maximum vertical strains. In both cases, the permeability is isotropic and obeys the Kozeny-Carmen relation. Figure 3 shows the traction on the top surface when $\gamma / L_{o}=0.005$ and $t_{o}=2.0$. We purposely apply a very small displacement on the top such that the infinitesimal assumption is valid. Because only a small amount of vertical strain is developed in the numerical specimen, the material deforms in geometrically linear regime, and the constitutive response is approximately linear. As a result, both the small and large strain formulations match the analytical solution in [53] as expected.

On the other hand, as we repeat the simulation with higher maximum displacement, $\gamma / L_{o}=1 \%$, $5 \%, 7.5 \%$, and $10 \%$ and lower loading rate $t_{o}=30$, the difference among the solutions obtained by the small strain FEM, the large strain FEM, and the analytical solution becomes apparent. First, we observe that the small strain solutions consistently predict lower peak traction but roughly the same steady-state traction compared with the analytical solution. This difference owes to the fact that the permeability obeys the Kozeny-Carmen relation in both large and small strain finite element models but remains constant in the analytical solution. As a result of the porosity dependence introduced by the Kozeny-Carmen relation, the permeability reduces heterogeneously when the displacement is applied on the top surface, as shown in Figure 4. After the maximum displacement is reached, the top surface is held, and stress relaxation occurs until the homogeneous drained response is reached, as shown in Figure 5. Because the permeability in the finite element models is reduced by the Kozeny-Carmen 


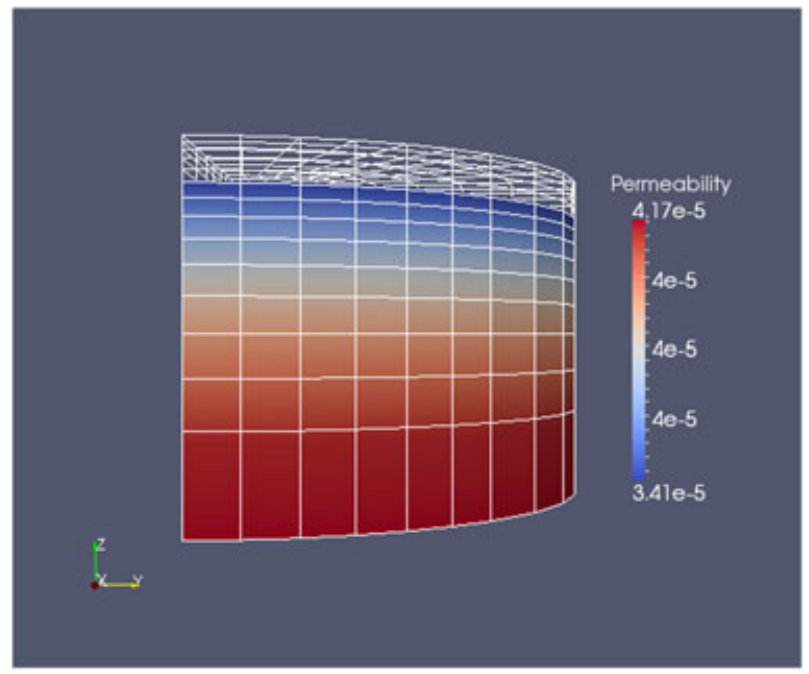

Figure 4. Permeability of the fully saturated specimen at the peak traction. The undeformed mesh is plotted in white.

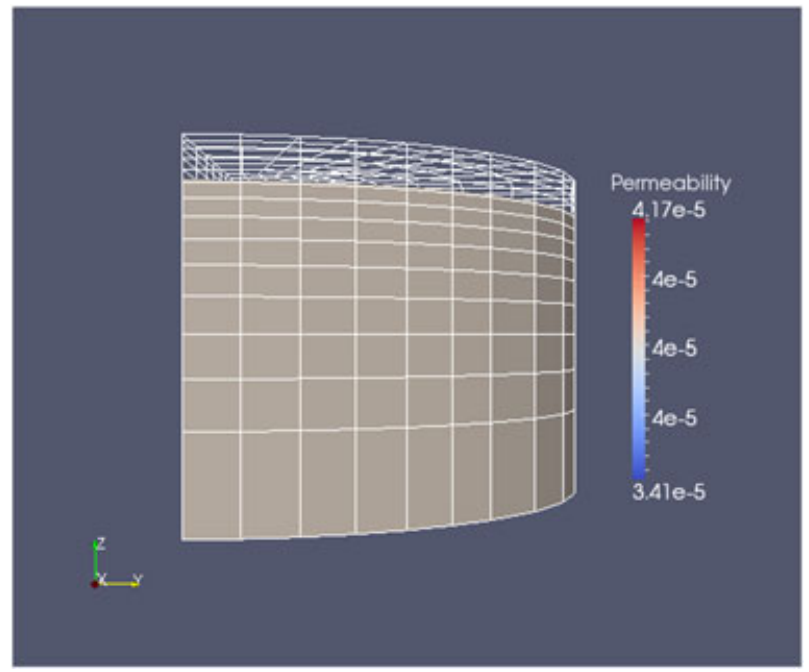

Figure 5. Permeability of the fully saturated specimen at the steady state. The undeformed mesh is plotted in white.

relation, it takes longer time for the seepage flow to reach steady state, and therefore, the steady state of the finite element models is delayed. Nevertheless, because the steady-state solution does not depend on permeability and the drained elastic response is linear, the traction predicted by the small strain finite element model matches the analytical solution.

Meanwhile, the large strain finite element model consistently predicts higher peak and steady-state tractions than the small strain and analytical counterparts. This trend becomes more significant for specimens undergoing higher maximum vertical strain, because they are further from the geometrical and material linear regime, as shown in Figure 6.

\section{Remark 8}

The permeability data are stored at integration points of the finite element. To generate the permeability contour in Figures 4 and 5 , a global $L_{2}$ projection is performed to map integration point data to the nodes and then use basis functions of the finite element to interpolate the scalar permeability field. Interested readers are referred to [31] for details. 


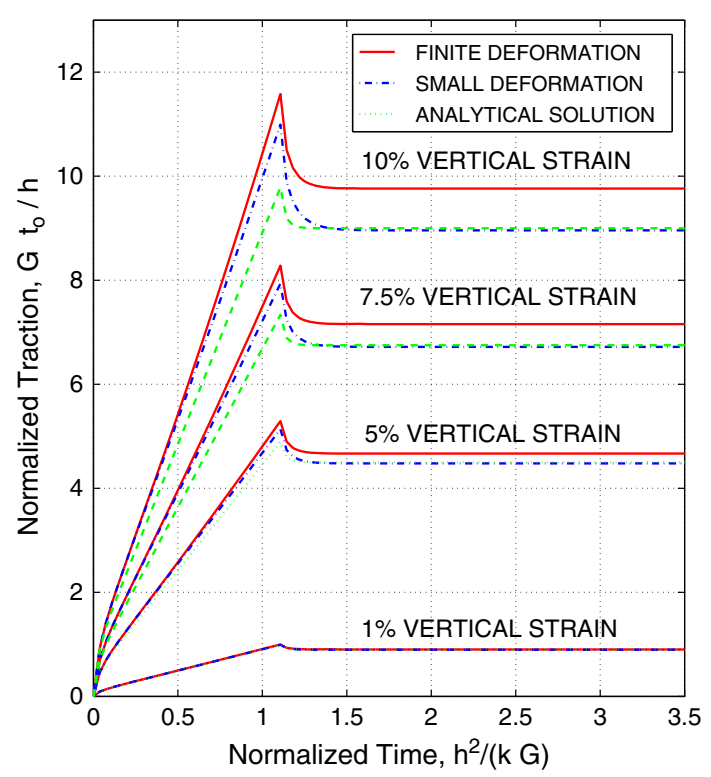

Figure 6. Dimensionless traction response obtained via stabilized finite element method and analytical solution at the top of the fully saturated specimen $\left(\gamma / L_{o}=1 \%, 5 \%, 7.5 \%, 10 \%\right.$ and $\left.t_{o}=30.0\right)$.
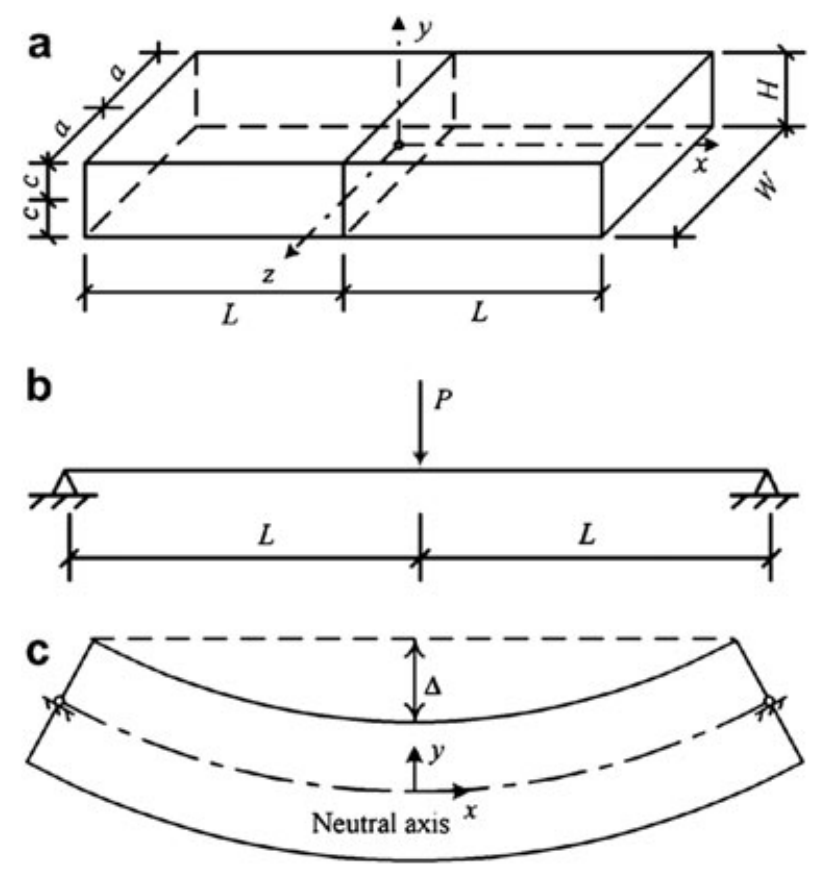

Figure 7. Bending of poroelastic beam: (a) dimensions of the three-dimensional model; (b) three-point bending scheme; and (c) longitudinal section. Figure reproduced from [56].

\subsection{Bending of a slender poroelastic beam}

We simulate the beam-bending problem in [56] for three reasons-(i) to compare the finite element simulations against the analytical solution when an assumed deformation gradient is used; (ii) to check whether the stabilization schemes are capable of eliminating spurious oscillation modes in multidimensional problems; and (iii) to verify whether volumetric locking can be overcome by the assumed deformation gradient formulation. 
Consider that a long and slender beam composed of poroelastic material is subjected to a three-point bending load where fluid is drained through the top, bottom, and lateral surfaces, as shown in Figure 7.

By assuming that the shear and transverse stresses are both small, Scherer, Prevost, and Wang [56] showed that the small strain load relaxation function can be approximated by

$$
\frac{P\left(\tau_{u}\right)}{P_{u}}=\frac{1+v}{1+v_{u}}+\frac{v_{u}-v}{1+v_{u}} S_{1}\left(\tau_{u}\right) S_{2}\left(\frac{c}{a}, \tau_{u}\right)
$$

where $P_{u}$ is the initial load required to hold the deflection at the undrained limit and $P$ is the applied load required to hold the same deflection of the beam. $v_{u}$ and $v$ are the undrained and drained Poisson ratios that are related via

$$
v_{u}=\frac{3 v+S(1-2 v) B}{3-S(1-2 v) B}, \quad S=\frac{B M}{K+B^{2} M}
$$

$\tau_{u}$ is the dimensional time that reads

$$
\tau_{u}=\frac{E k t}{2 c^{2}(1+v)(1-2 v)}
$$

$S_{1}(\tau)$ and $S_{2}\left(\frac{c}{a}, \tau\right)$ are the decay functions that read

$$
S_{1}(\tau)=\sum_{m=1}^{\infty} \frac{6}{(a \pi)^{2}} \exp \left(-\tau_{u}(m \pi)^{2}\right)
$$

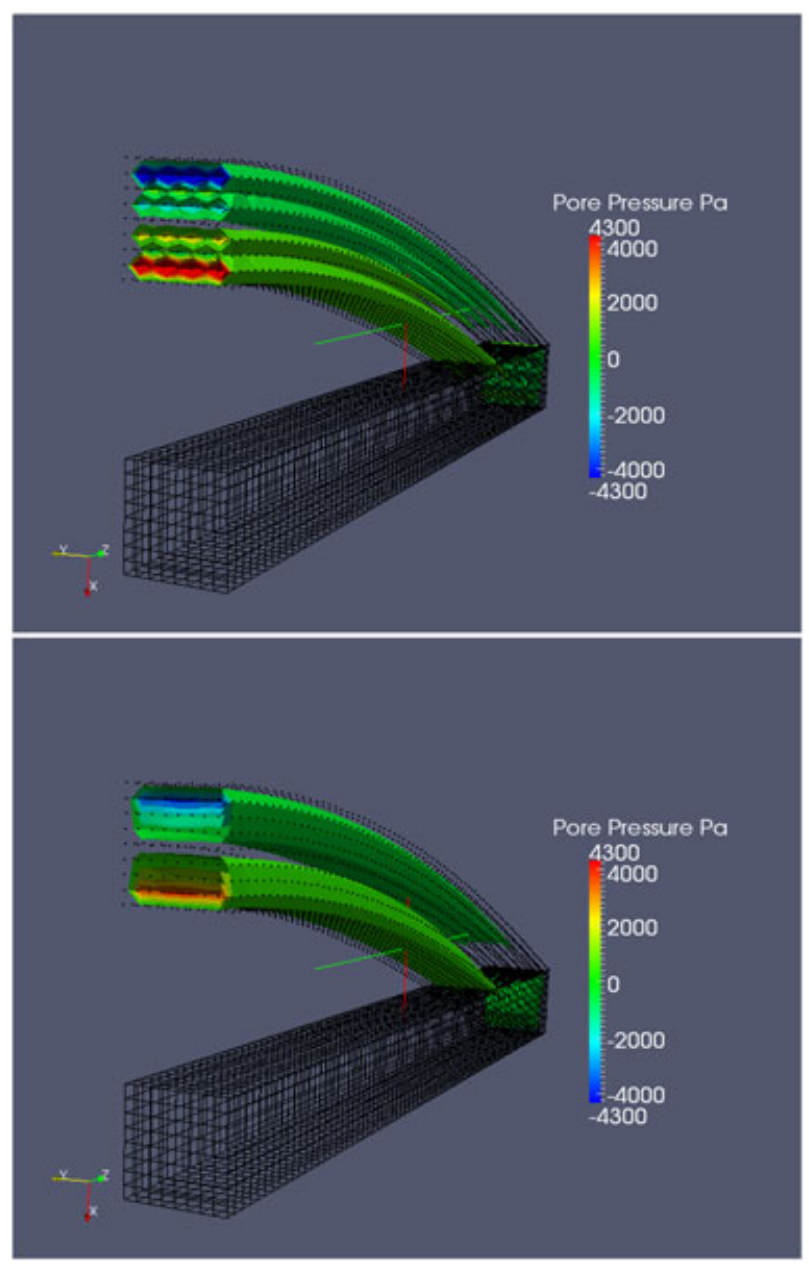

Figure 8. Contour of pore pressure for pure bending problem at $t=0.00002 \mathrm{~s}$ without (top) and with (bottom) the stabilization procedure. Displacement scaled by 5 . 


$$
S_{2}\left(\frac{c}{a}, \tau_{u}\right)=\sum_{n=1}^{\infty} \frac{2}{b_{n}^{2}} \exp \left(-b_{n}^{2} \frac{c^{2}}{a^{2}} \tau_{u}\right)
$$

where $b_{n}=(2 n-1) /(2 \pi)$. This pure bending problem is replicated via finite element simulation with and without the stabilization procedure. Because of symmetry, only half of the beam is meshed. The following material parameters are used: $E=1 \mathrm{MPa}, v=0.4, K_{s}=1 \mathrm{GPa}, K_{f}=0.1 \mathrm{GPa}$, initial isotropic hydraulic conductivity $=k / \mu=6.1 \times 10^{-11} \mathrm{~m}^{2} / \mathrm{Pa} \mathrm{s}$, and initial porosity $=0.4$. Body forces are neglected. The dimensions of the square beam are $L=0.1 \mathrm{~m}$ and $a=c=0.005 \mathrm{~m}$, and the prescribed deflection is $0.0025 \mathrm{~m}$. The finite element domain consists of 5000 equal size eight-node hexahedral finite elements. This mesh is much coarser than the mesh used in [56] to test whether the stabilization scheme can eliminate spurious oscillation in the difficult scenario where element sizes are large relative to the diffusivity and discrete time step. A full backward Euler scheme is used for time integration.

Figure 8 shows the contour of pore pressure at $t=0.00002 \mathrm{~s}$ for the beam deflection simulations. In both simulations, a full assumed deformation gradient is used, that is, $\alpha=0$. The resultant pore pressure depicted in Figure 8 clearly shows that oscillation occurs in the simulation with equal order discretization but does not occur if the stabilization procedure is used.

To illustrate how the assumed deformation gradient formulation overcomes the volumetric locking problem, we conduct a refinement study for the pure bending poroelastic beam problem alluded to
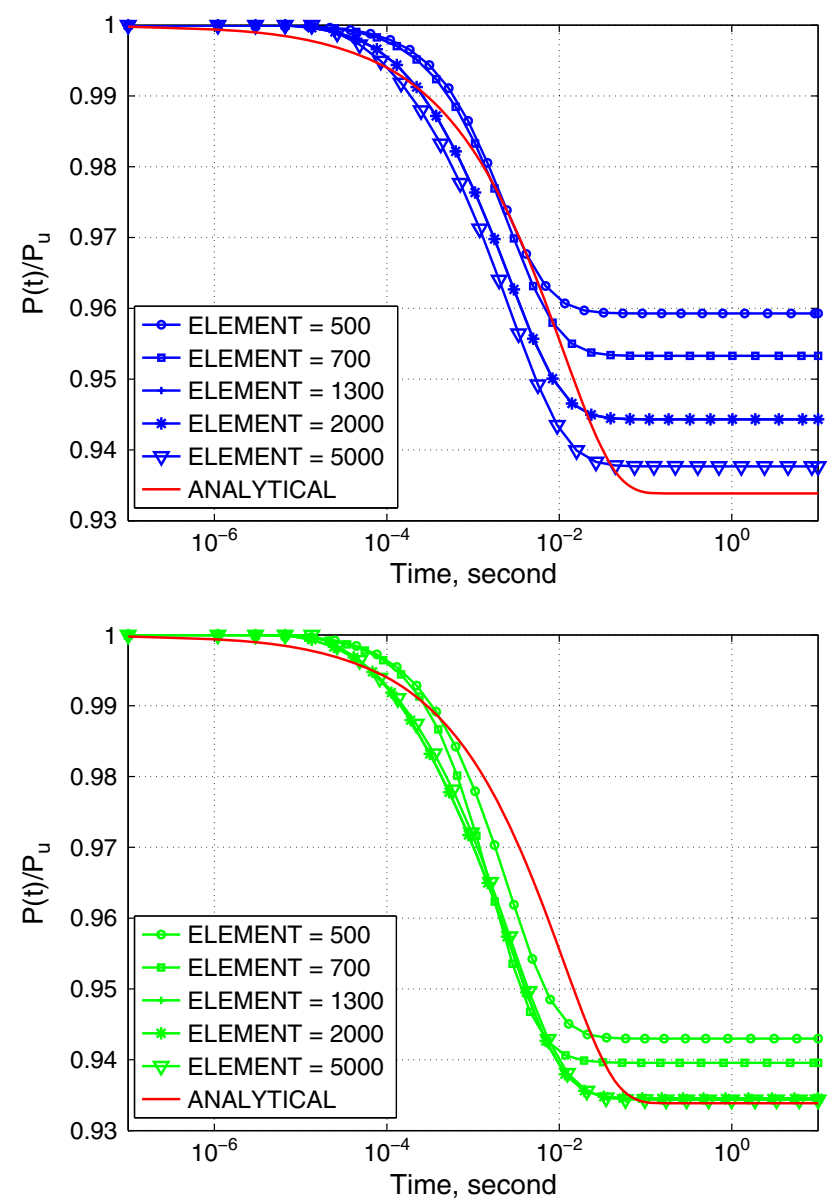

Figure 9. Load relaxation curve versus time for meshes with various refinement levels obtained from full deformation gradient simulations (top, in blue), assumed deformation gradient simulation (bottom, in green color), and analytical solution with constant coefficients (in red color). 
earlier with various finite element meshes composed of 400-20,000 elements of uniform sizes. The results with full and assumed gradients are compared with the analytical solutions in Figure 9. Clearly, the relaxation load obtained via the assumed deformation gradient converges to the asymptotic solution $(1+v) /\left(1-v_{u}\right)$ with around 2000 elements, whereas the full deformation gradient simulation does not converge to the analytical solution even with 5000 elements. This result demonstrates that the assumed deformation gradient formulation exhibits improved element performance over the full deformation gradient simulation for this bending dominated problem. As pointed out in [56], the analytical solution is obtained under small deformation assumption, and by neglecting contributions from shear and transverse stresses, and the evolution of material parameters. Hence, discrepancies between analytical and numerical solutions are expected, although they should be relatively small.

\subsection{Plane strain footing problem}

We adopt a simplified version of the rigid footing problem in [25] to (i) to compare the mechanical and hydraulic responses obtained by full and assumed deformation gradient formulations after strain localization take places and (ii) test whether spurious pore pressure oscillation modes can be removed by the projection stabilization procedure.

The boundary value problem representing the installation of a rigid footing on a porous medium can be described as follows, assuming a plane strain condition holds. Consider a 5-m long rigid footing placed on a homogeneous fully saturated square soil deposit with side length $=10 \mathrm{~m}$. A vertical

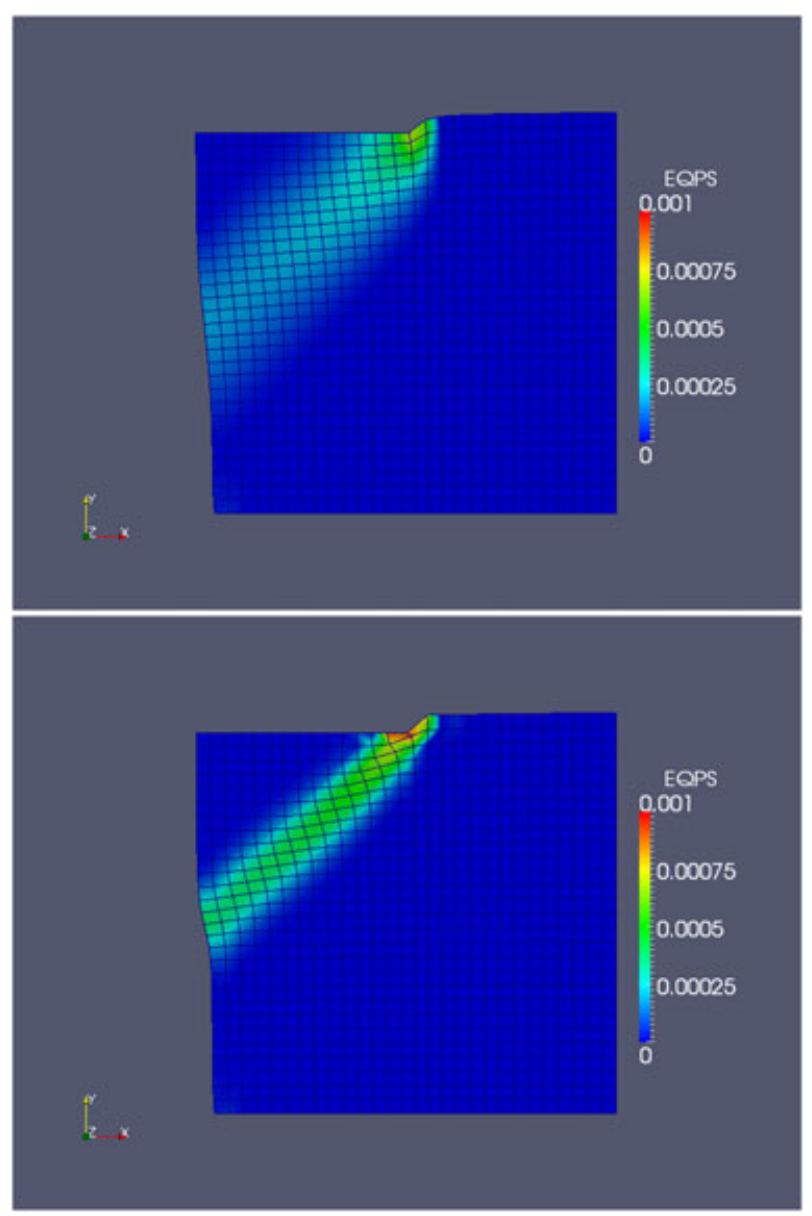

Figure 10. Equivalent plastic strain obtained from full deformation gradient (top) and assumed formation gradient (bottom) at $t=10 \mathrm{~s}$. Poisson ratio $=0.4$. Displacement is multiplied by 500. Equivalent plastic strain field is projected from interpolation points to nodes via global $L_{2}$ projection algorithm in [31]. 
displacement is prescribed on the soil underneath the rigid footing at a constant rate $0.0001 \mathrm{~m} / \mathrm{s}$. Displacement on the right and bottom of the soil deposit is restricted. Drainage of water is allowed only through the unloaded part of the top surface of the soil deposit.

The following material parameters are used: $E=2 \mathrm{GPa} K_{s}=50 \mathrm{GPa}, K_{f}=2 \mathrm{GPa}$, initial isotropic hydraulic conductivity $=k / \mu=1.0 \times 10^{-14} \mathrm{~m}^{2} / \mathrm{Pa} \mathrm{s}$, and initial porosity $=0.4$. The plastic response of the material is captured via a perfectly plastic J2 model with yield strength $\sigma_{Y}=100 \mathrm{kPa}$ and hardening modulus $H=0$. An associative flow rule is used. Body forces are neglected. The finite element mesh consists of 1089 hexahedral elements (33 elements for each side and one element depth). Full backward Euler time integration is used with constant time step $=0.25 \mathrm{~s}$.

To compare the difference between the full and assumed deformation gradient formulations, we first conduct the footing simulation with Poisson ratio $=0.4$. Notice that for $\mathrm{J} 2$ perfectly plastic material, the plastic flow is isochoric and hence vulnerable to locking phenomena. Figure 10 compares the equivalent plastic strain obtained from the full and assumed deformation gradients at $10 \mathrm{~s}$ after the loading. In both cases, shear bands form after the onset of plastic deformation. However, the shear band appears to be narrower when assumed deformation gradient is used.

Figure 11 compares the pore pressure obtained from the full and assumed deformation gradient formulations. Interestingly, we obtained a narrow negative pore pressure zone near the edge of the footing in the full deformation gradient simulation but not in the assumed deformation gradient formulation. This negative pore pressure zone is possibly due to the locking of the volumetric deformation at the edge where significant shear strain develops.

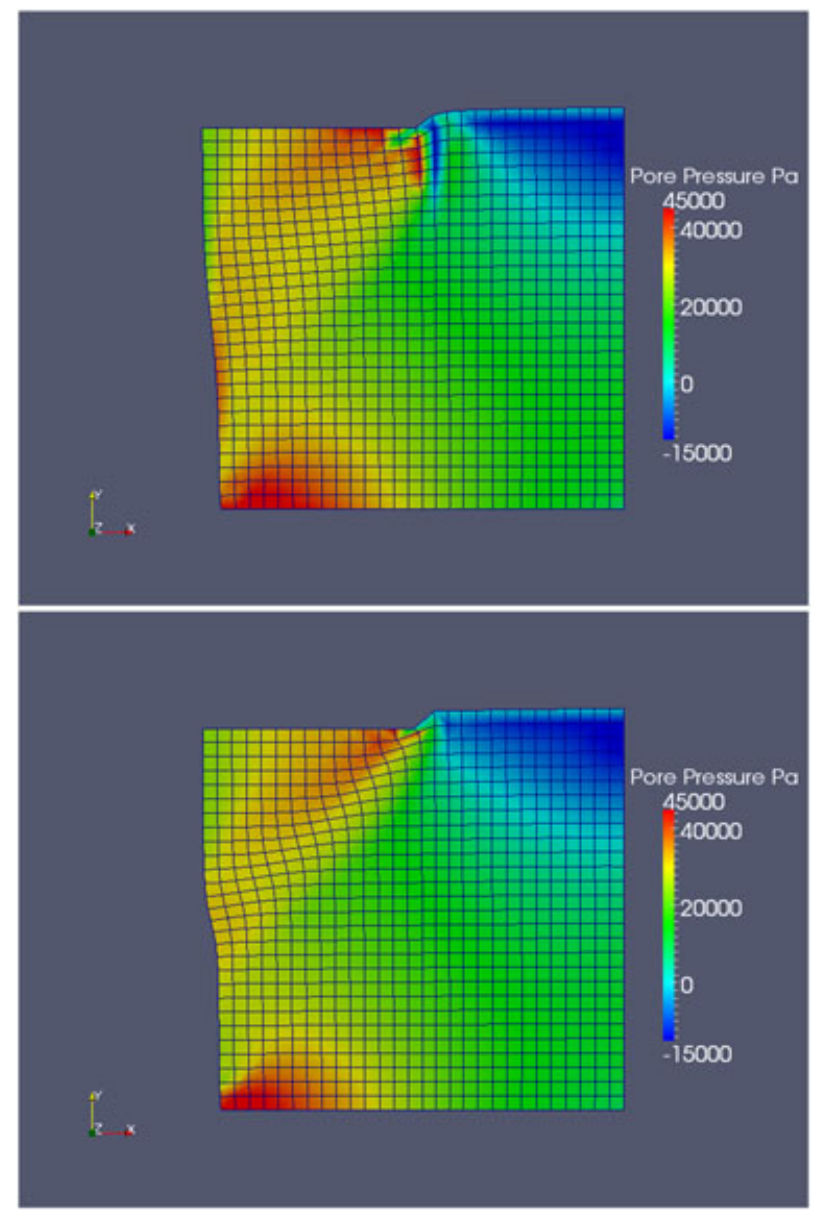

Figure 11. Pore pressure field obtained from full deformation gradient (top) and assumed formation gradient (bottom) at $t=10 \mathrm{~s}$. Poisson ratio $=0.4$. Displacement is multiplied by 500 . 
Figure 12 compares the pore pressure and displacement time histories of the node at the middle of the left vertical surface. Four simulations are conducted with the following settings: (i) stabilization and F-bar enabled (red); (ii) stabilization enabled (blue); (iii) F-bar enabled (purple); and (iv) standard Galerkin method (cyan). The reason we include this example is to show that the stabilized and F-bar methods do not cause unusual behavior. In fact, the pore pressure time histories of the four simulations are identical in the first $4 \mathrm{~s}$ and only differ after formation of the shear band at $t=4 \mathrm{~s}$. Although employing stabilization and/or the assumed deformation gradient method may lead to lower peak pore pressure as expected, the differences between the four simulations are insignificant. On the other hand, all four simulations predict almost identical displacement time histories.

\subsection{Circular footing}

The circular footing problem is used to test the performance of the stabilization scheme with a nonuniform mesh in three dimensions. In this problem, a rigid circular footing with radius $r=3 \mathrm{~m}$ is placed on the top of a cylindrical soil deposit with $30 \mathrm{~m}$ radius and $30 \mathrm{~m}$ height. A ramp function
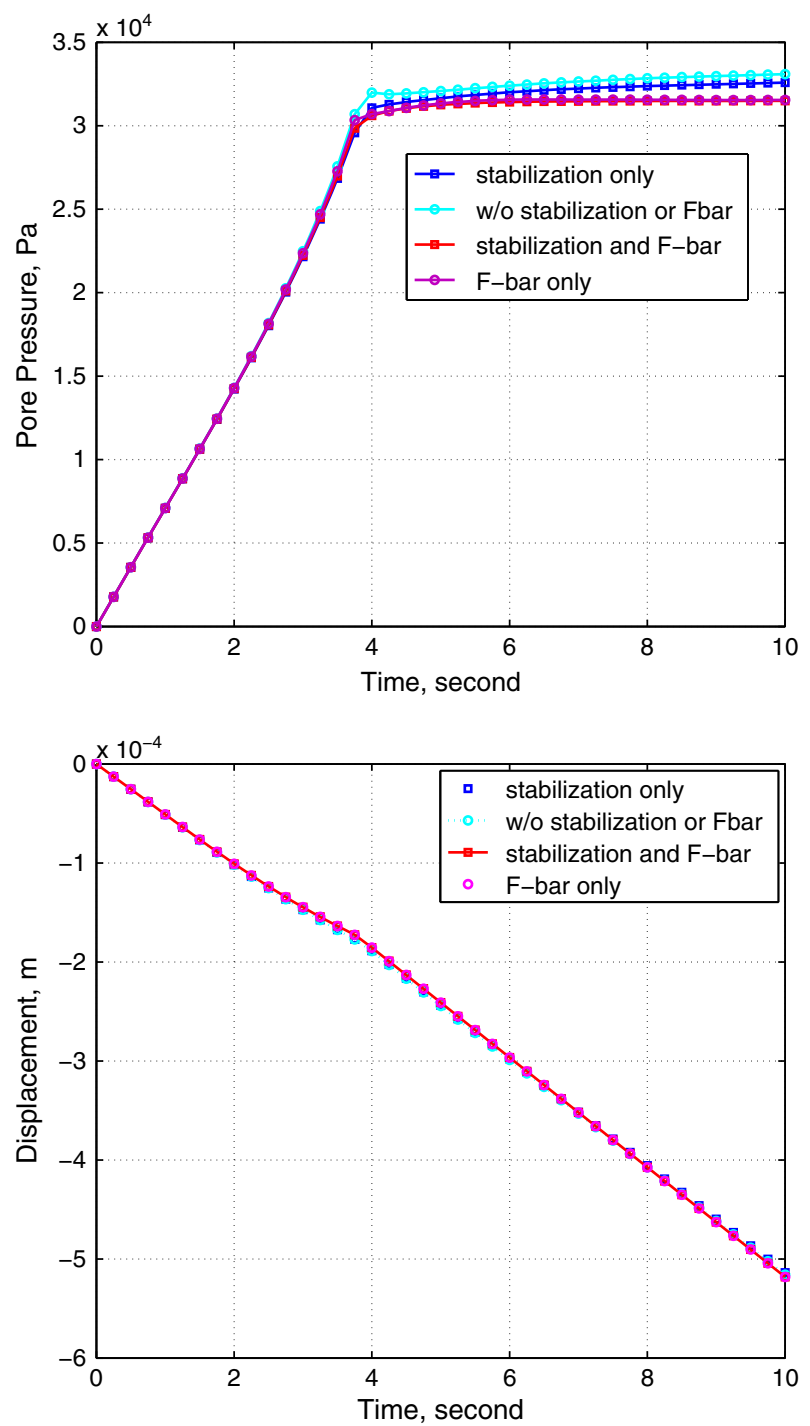

Figure 12. Pore pressure (top) and displacement (bottom) time histories for the elasto-plastic footing example. Results are obtained from stabilized F-bar (red), stabilized (blue), F-bar (purple), and standard Galerkin (cyan) formulations. 


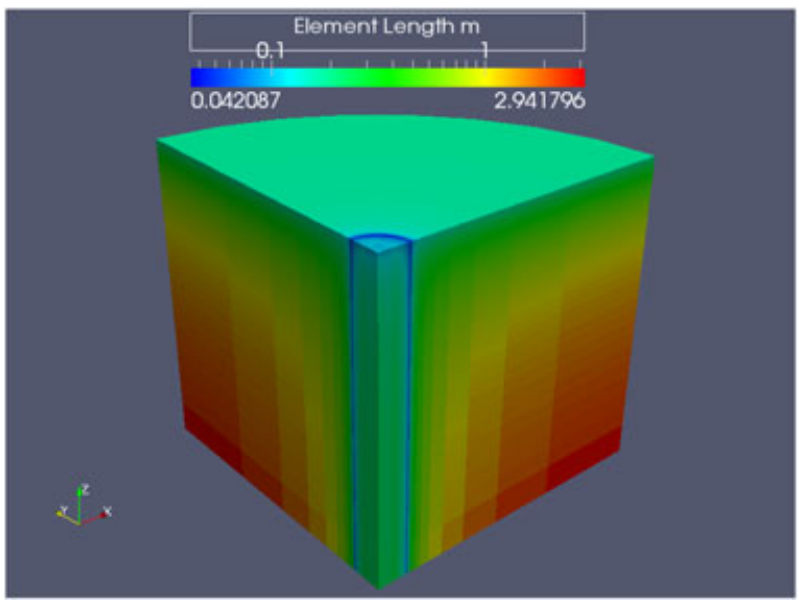

Figure 13. Finite element mesh used for the circular footing simulation. Element length calculated via (79).
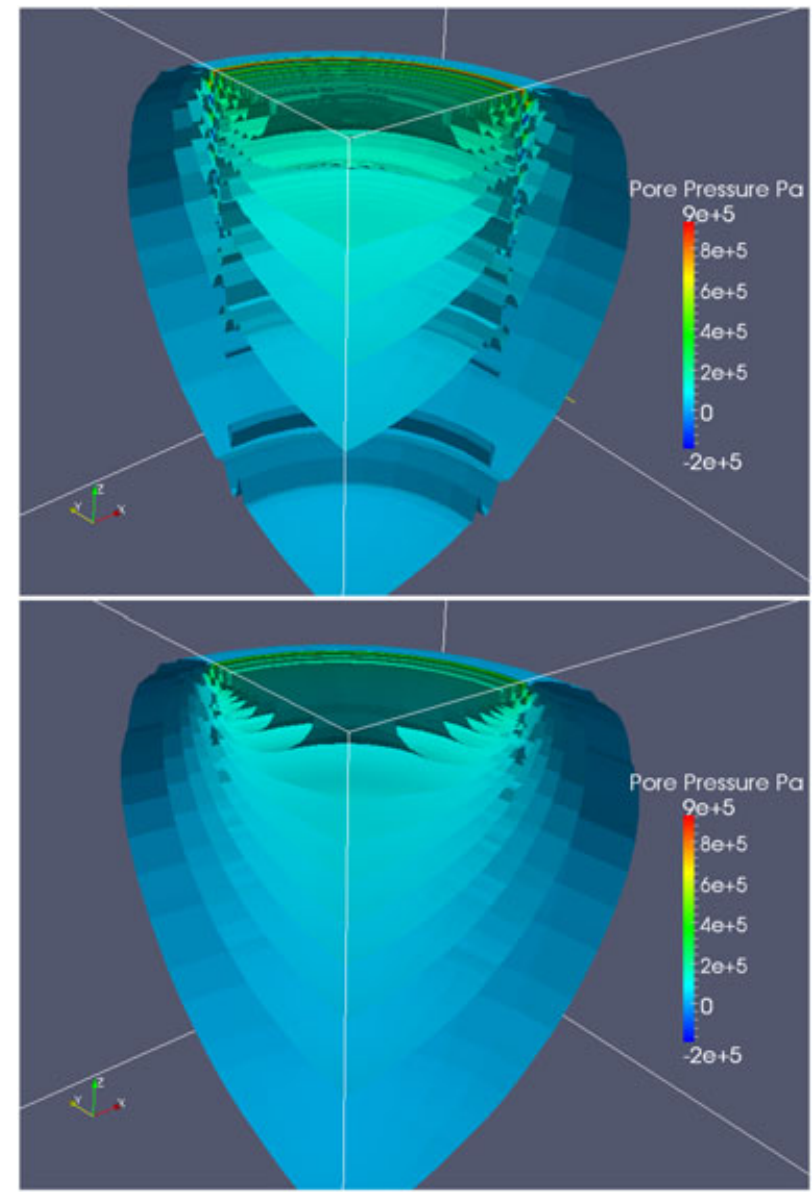

Figure 14. Isosurfaces of pore pressure field of the circular elasto-plastic footing at time $=0.005 \mathrm{~s}$ obtained from the standard F-bar (top) and stabilized F-bar (bottom) schemes, low hydraulic conductivity case, with $k / \mu=8.333 \times 10^{-21} \mathrm{~m}^{2} / \mathrm{Pa}$ s. 
is used to prescribed vertical displacements on the soil underneath the circular footing at a constant rate $0.2 \mathrm{~m} /$ day. The assumed deformation gradient is used for both stabilized and standard formulations. The intention is the application of loads on a porous medium with very low permeability and small time step to ensure the onset of spurious oscillatory modes in the standard formulation and then to check whether the stabilized formulation can completely eliminate them in this difficult situation. Because of symmetry, only a quarter of the domain is meshed. The material parameters of the soil deposit are as follows : $E=100 \mathrm{MPa} K_{s}=5 \mathrm{GPa}, K_{f}=2 \mathrm{GPa}$, initial isotropic hydraulic conductivity $=k / \mu=8.333 \times 10^{-21} \mathrm{~m}^{2} / \mathrm{Pa} \mathrm{s}$, and initial porosity $=0.5$. The plastic response of the material is captured via a perfectly plastic $\mathrm{J} 2$ model with yield strength $\sigma_{Y}=500 \mathrm{kPa}$ and hardening modulus $H=0$. An associative flow rule is used, and body forces are neglected.

A nonuniform mesh is used with very fine elements near the edge and coarser elements in the far field, as shown in Figure 13. This discretization constitutes a worst case scenario in which permeability is low, time step is fine, and mesh is nonuniform. Figure 14 compares the results at time $t=0.005 \mathrm{~s}$. For such a low permeability and small time step, the standard formulation leads to spurious oscillation as expected. In particular, oscillations are observed near the edge between fine and coarse elements. As shown in Figure 14, oscillation modes have been significantly suppressed in the stabilized simulation. Nevertheless, spike patterns can be observed in the stabilized solution near the tip of the footing. It appears that the sharp pore pressure gradient triggered by the ground movement is so large in the nonuniform mesh that the stabilization parameter estimated from 1D uniform grid is unable to provide

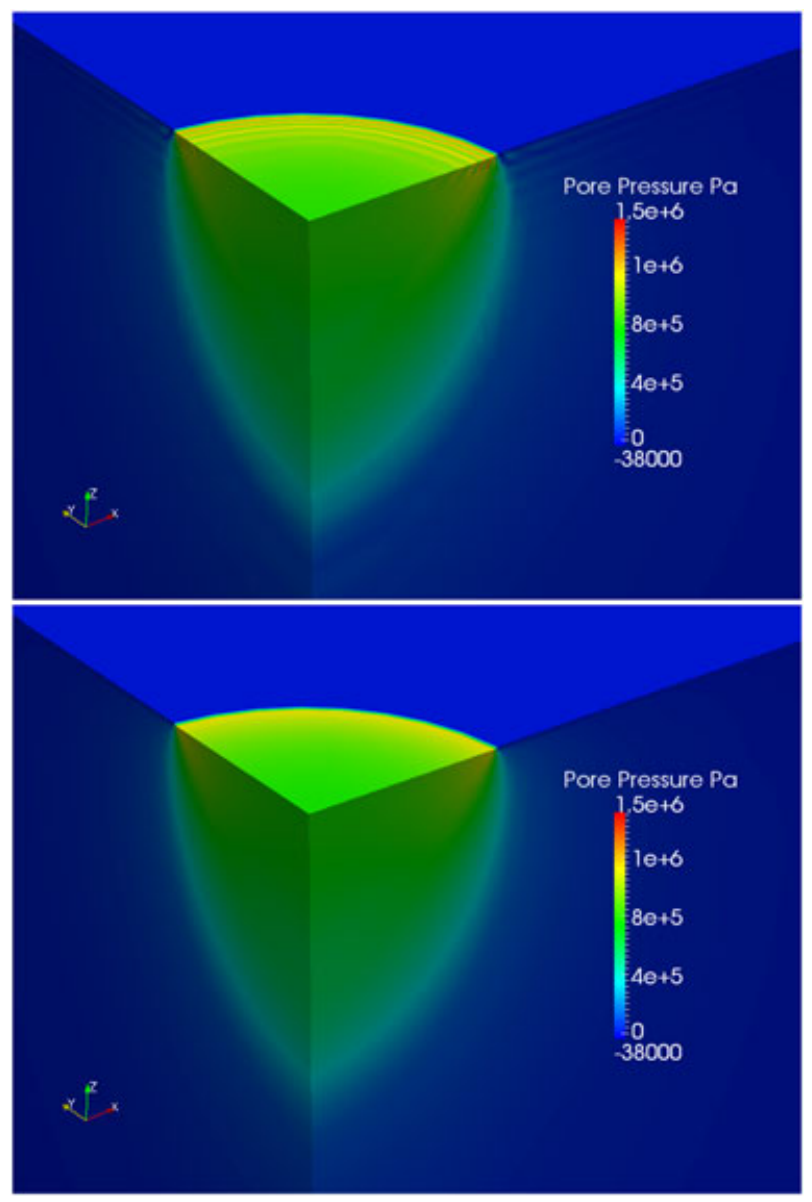

Figure 15. Contours of the pore pressure field of the circular elasto-plastic footing at time $=20,000 \mathrm{~s}$ obtained from the standard F-bar (top) and stabilized F-bar (bottom) schemes, low hydraulic conductivity case, with $k / \mu=8.333 \times 10^{-21} \mathrm{~m}^{2} / \mathrm{Pa} \mathrm{s}$. 
sufficient relaxation to completely remove the oscillation. Similar unstable pore pressure profiles were found in [18] where both Q9P4 Taylor-Hood elements and stabilized Q4P4 elements lead to oscillatory pore pressure profiles near a sharp gradient in a nonuniform mesh. In [18], the stabilization parameter was increased by a factor of 25 to completely eliminate the oscillatory pattern. Presumably, this shortcoming can also be remedied if a higher stabilization parameter is used in the present case. How to estimate the optimal value for the stabilization parameter in a nonuniform mesh with shape pore pressure gradient and small time step is currently unknown but will be considered in future studies.

Figure 15 presents the contour of the pore pressure field at time $=20,000 \mathrm{~s}$ obtained from the stabilized F-bar and standard formulations. In this case, we notice that the stabilization scheme is able to eliminate the pore pressure oscillation. This is because the diffusion process may smoothen the pore pressure profile over time, provided that the hydraulic conductivity tensor is symmetric and positive definite.

Figure 16 compares the equivalent plastic strain at time $=20,000 \mathrm{~s}$. At this instant, considerable plastic strain has developed inside a localized zone. We found no discrepancy to exists in the mechanical responses between the standard and stabilized formulations at 20,000 s. This result indicates that the stabilization scheme does not introduce any artificial behavior other than filtering out the oscillation pattern that arises because of lack of $H^{1}$ stability.

Figure 17 presents the pore pressure time histories at the center (top) and tip (bottom) of the circular footing. Whereas the two pore pressure time histories obtained from the stabilized and standard F-bar

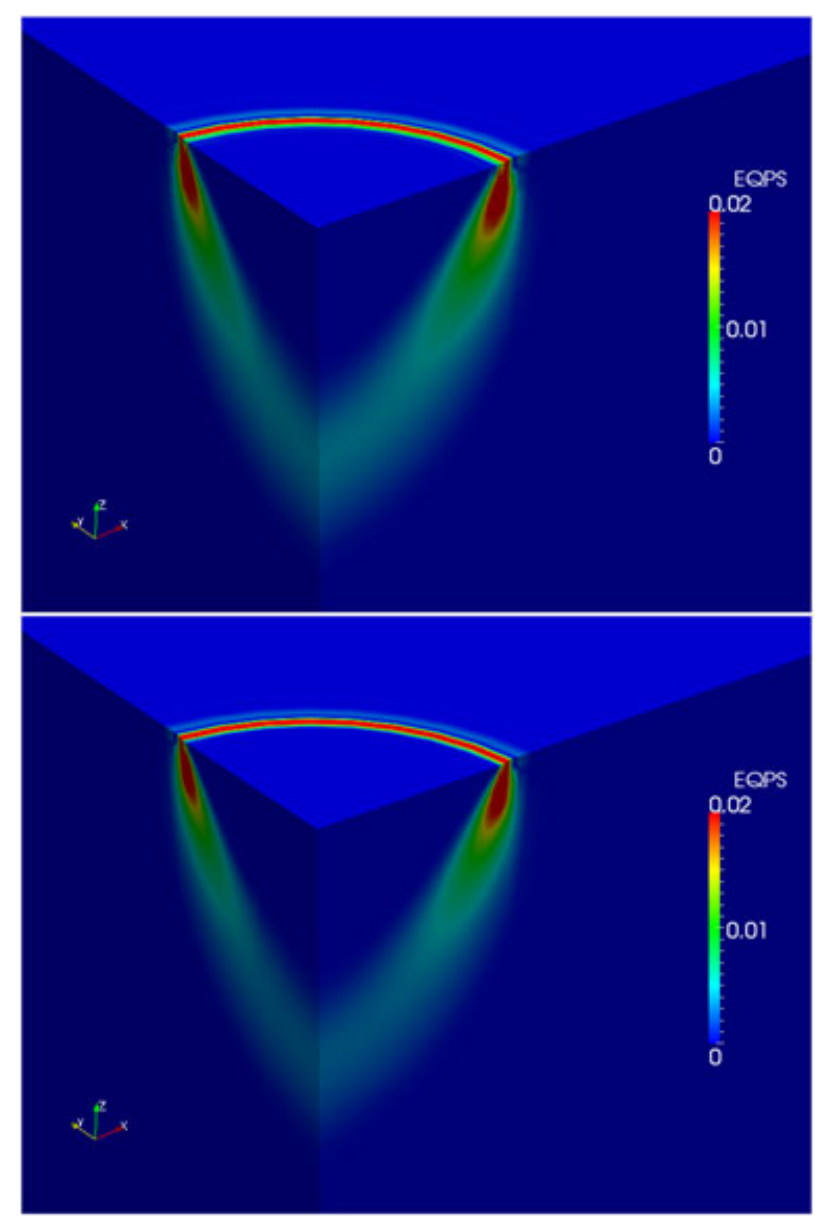

Figure 16. Contours of the equivalent plastic strain of the circular elasto-plastic footing at time $=20,000 \mathrm{~s}$ obtained from standard F-bar (top) and stabilized F-bar (bottom) schemes, low hydraulic conductivity case, with $k / \mu=8.333 \times 10^{-21} \mathrm{~m}^{2} / \mathrm{Pa} \mathrm{s}$. 

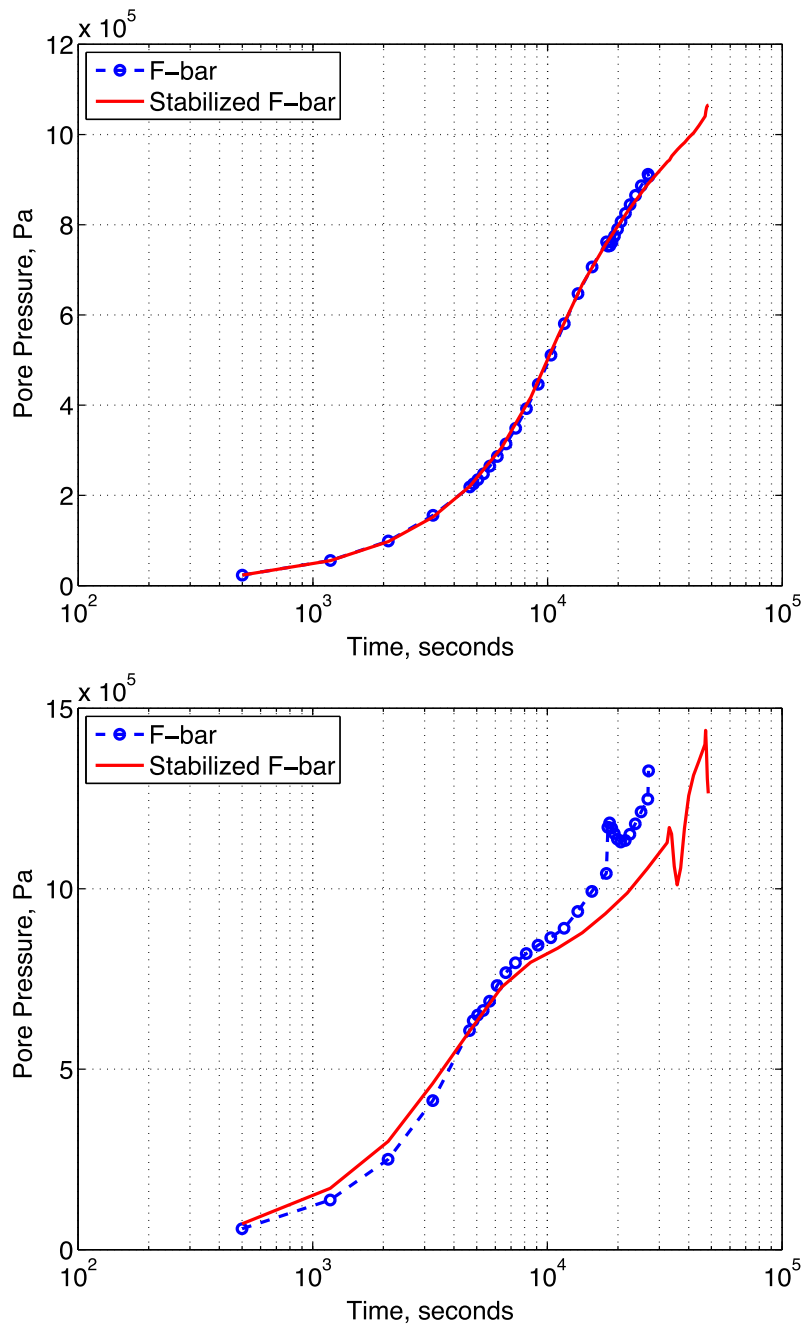

Figure 17. Time histories of pore pressure at the center (top) and tip (bottom) of the circular footing, low hydraulic conductivity case, with $k / \mu=8.333 \times 10^{-21} \mathrm{~m}^{2} / \mathrm{Pa}$.

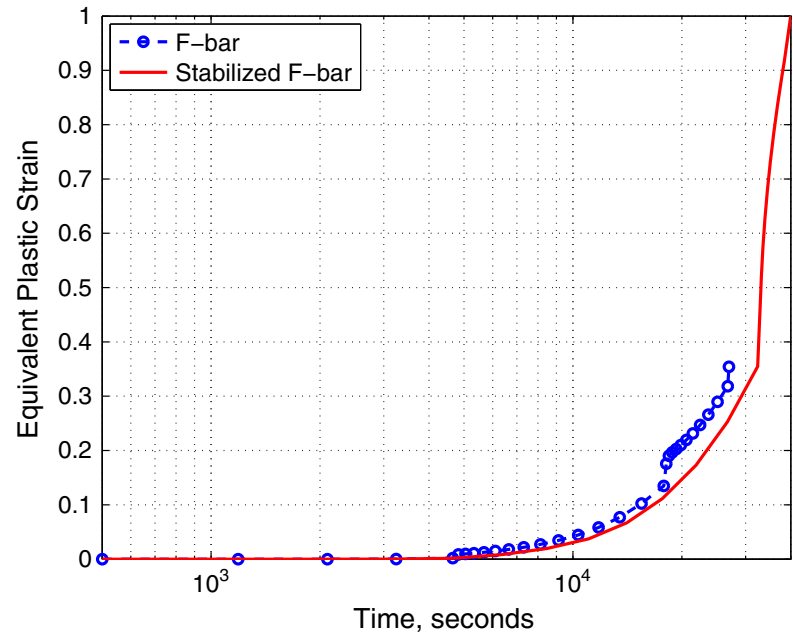

Figure 18. Time histories of equivalent plastic strain and tip of the circular footing (bottom), low hydraulic conductivity case, with $k / \mu=8.333 \times 10^{-21} \mathrm{~m}^{2} / \mathrm{Pa} \mathrm{s}$. 
schemes match quite well at the center, they differ at the tip where the oscillation takes place as shown in Figures 14 and 15. This finding is consistent with [57], in which the author proved that the standard Galerkin method tends to underdiffuse solutions in the presence of sharp gradients but may perform well in the absence of it.

Notice that the pore pressure at the tip of the footing predicted by the standard scheme tends to be lower before the perfectly plastic yielding but higher after plastic strain accumulation. This switch from undershooting to overshooting occurs at the onset of yielding, as shown in Figure 18. In the postyielding regime, the equivalent plastic strain in the standard scheme is higher than that of the stabilized scheme, and this trend tends to be more significant as the amount of load increased with time.

Finally, we conduct simulations for the same problem but increase the initial isotropic hydraulic conductivity from $k / \mu=8.333 \times 10^{-21}$ to $k / \mu=8.333 \times 10^{-11} \mathrm{~m}^{2} / \mathrm{Pa}$ s to test whether the stabilization scheme generates significant diffusion or unusual behavior when stability is expected for both stabilized and standard F-bar methods. Figure 19 presents the pore pressure obtained from the stabilized F-bar and standard Galerkin simulations at 20,000 s. We found that the two simulations lead to almost identical solutions and that no unusual behavior is observed in the tip of the footing. Figure 20 presents the time histories of the pore pressure at the center and tip of the footing. Again, we observed no noticeable difference between the standard and stabilized F-bar methods. The same conclusion is drawn from Figure 21 in which the equivalent plastic strain at the tip obtained by stabilized and standard F-bar schemes exhibits identical time histories.

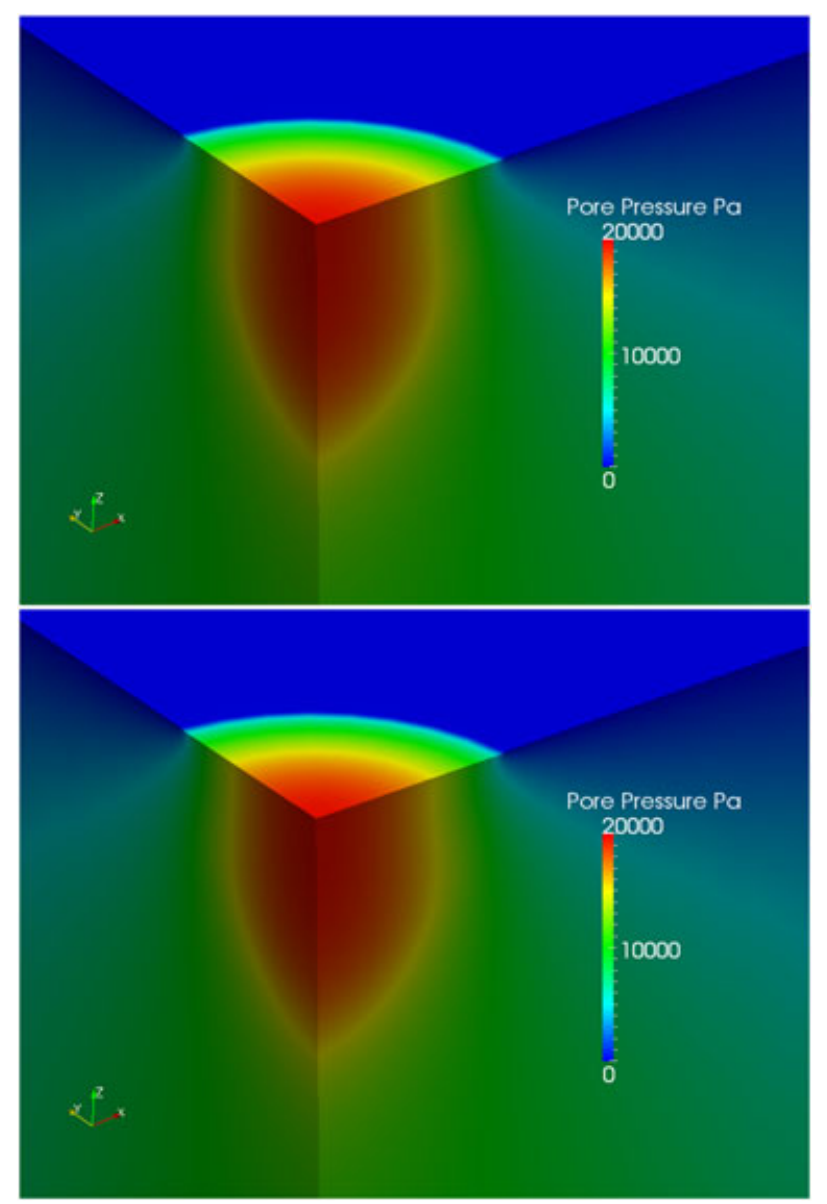

Figure 19. Contours of the pore pressure field of the circular elasto-plastic footing at time $=20000 \mathrm{~s}$ obtained from standard F-bar (top) and stabilized F-bar (bottom) schemes, high hydraulic conductivity case, with $k / \mu=8.333 \times 10^{-11} \mathrm{~m}^{2} / \mathrm{Pa} \mathrm{s}$. 

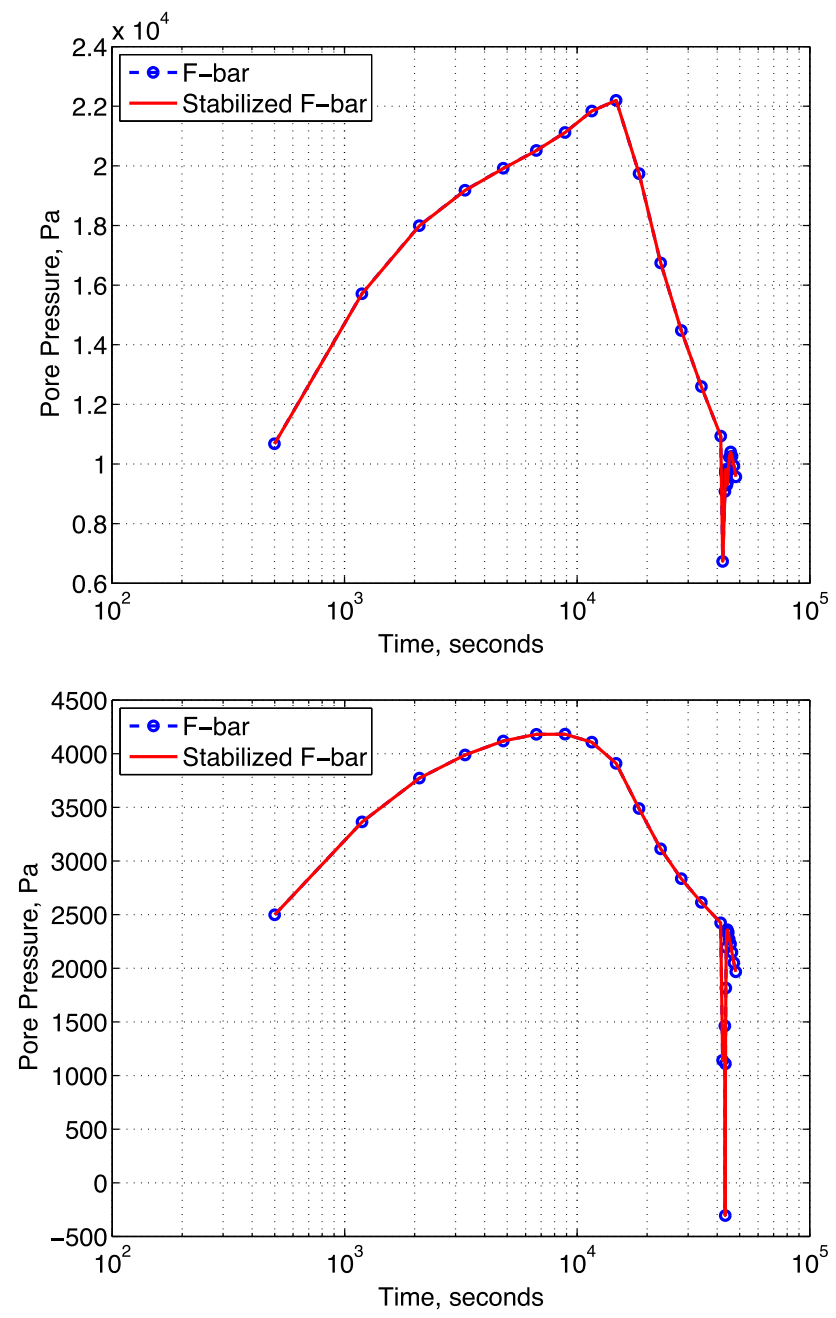

Figure 20. Time histories of pore pressure at the center (top) and tip (bottom) of the circular footing, high hydraulic conductivity case, with $k / \mu=8.333 \times 10^{-11} \mathrm{~m}^{2} / \mathrm{Pa} \mathrm{s}$.

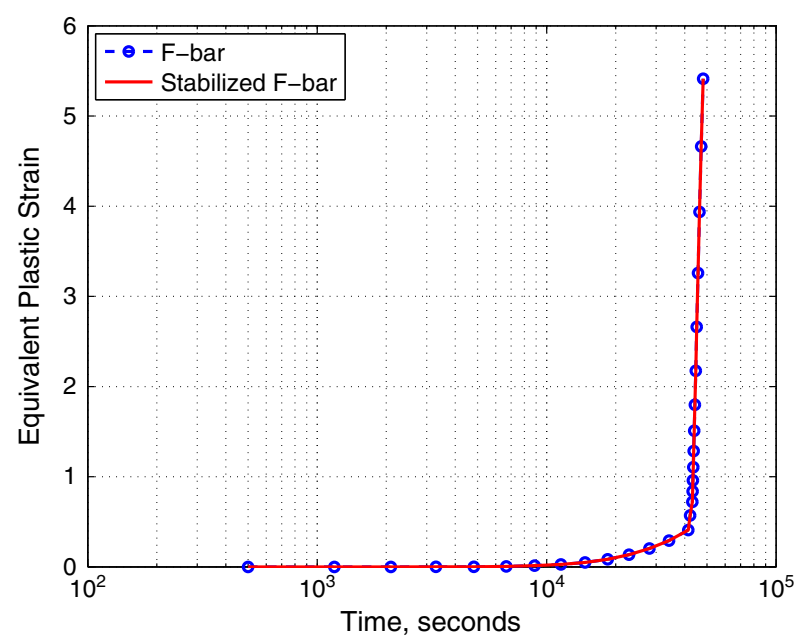

Figure 21. Time histories of equivalent plastic strain and tip of the circular footing (bottom), high hydraulic conductivity case, with $k / \mu=8.333 \times 10^{-11} \mathrm{~m}^{2} / \mathrm{Pa} \mathrm{s}$. 
The agreement between the standard and adaptively stabilized Galerkin methods indicates that the stabilization term does not introduce noticeable overdiffusion. In fact, the arctangent term in (77) effectively turns off the stabilization scheme whenever it is not needed. As a result, there is no need to manually adjust the stabilization parameter for the high diffusivity case.

\section{CONCLUSION}

In this work, we propose the usage of an adaptively stabilized scheme on an assumed deformation gradient poromechanics formulation to deliver stable, locking-free numerical solutions. By using equal-order integration, we establish monolithically coupled poromechanics finite element models with less degrees of freedom, fewer integration points, and simpler data structures but without the necessity to design complex splitting I/O algorithms required for sequential coupling schemes [9].

We show that the $L_{2}$ projection-based stabilization scheme proposed in [18] can be recast as a penalty energy formulation in which spurious pore pressure modes are filtered out by the penalty energy functional. Using this as our starting point, we apply Harari's condition [36] to adaptively estimate the optimal value for the stabilization parameter. Thus, no tuning and additional input are required from the users. By applying an assumed deformation gradient to the formulation, we improve the element performance of the stabilized formulation when locking may occur.

Numerical examples have demonstrated that the formulation with stabilized scheme and assumed deformation gradient is robust and leads to high-quality, locking-free solutions. In particular, the introduction of an assumed deformation gradient does not result in a negative impact on the stability of the solutions. Numerical studies indicate that problems with a fine mesh, high diffusivity, and large time step but without sharp pore pressure gradient tend to deliver more stable solutions. Meanwhile, problems with coarse mesh, low diffusivity, small time step, and sharp pore pressure gradients tend to exhibit spurious modes unless a stabilization scheme is used.

It is worthwhile to point out that the implementation of this formulation is significantly simplified via template programming and in particular the usage of $\mathrm{AD}$.

Because both locking and instabilities are commonly encountered in engineering applications for porous media, the stable and locking-free features demonstrated in this formulation are highly desirable. Future work will include further analysis of the formulation near boundaries with more advanced gradient-capturing techniques as those in [45] and the application of this formulation with more realistic constitutive models for both solid and fluid constituents (e.g., [58, 59]).

\section{ACKNOWLEDGEMENTS}

Thanks are due to Joshua A. White, Alejandro Mota, and James W. Foulk III for their helpful discussion and Eric Phipps for his significant contributions to Albany and the AD infrastructure. We also thank the reviewer for the constructive comment and suggestions. The Sandia National Laboratories is a multiprogram laboratory managed and operated by Sandia Corporation, a wholly owned subsidiary of Lockheed Martin Corporation, for the U.S. Department of Energy's National Nuclear Security Administration under contract DE-AC04-94AL85000.

\section{REFERENCES}

1. Biot MA. General theory of three-dimensional consolidation. Journal of Applied Physics 1941; 12:155-164.

2. Borja RI. On the mechanical energy and effective stress in saturated and unsaturated porous continua. International Journal of Solids and Structures 2006; 43:1764-1786.

3. Cowin SC, Doty SB. Tissue Mechanics. Springer: New York, N.Y, 2006.

4. Coussy O, Dormieux L, Detournay E. From mixture theory to Biot's approach for porous media. International Journal of Solids and Structures 1998; 35(34-35):4619-463.

5. Coussy O. Poromechanics. Wiley: New York, N.Y, 2004.

6. Jeremic B, Cheng Z, Taiebat M, Dafalias Y. Numerical simulation of fully saturated porous materials. International Journal for Numerical and Analytical Methods in Geomechanics 2008; 32(13):1635-1660.

7. Zienkiewicz OC, Chan AHC, Pastor M, Schrefler BA, Shiomi T. Computational Geomechanics with Special Reference to Earthquake Engineering. Wiley: New York, N.Y, 2001.

8. Mandel J. Consolidation des sols. Géotechnique 1953; 3:287-299. 
9. Kim J, Tchelepi HA, Juanes R. Stability and convergence of sequential methods for coupled flow and geomechanics: Fixed-stress and fixed-strain splits. Computer Methods in Applied Mechanics and Engineering 2011; 200:1591-1606.

10. Armero F. Formulation and finite element implementation of a multiplicative model of coupled poro-plasticity at finite strain under fully saturated conditions. Computer Methods in Applied Mechanics and Engineering 1999; 171:205-241.

11. Andrade JE, Borja RI. Modeling deformation banding in dense and loose fluid-saturated sands. Finite Element in Analysis and Design 2007; 43:361-383.

12. Bathe KJ. The inf-sup condition and its evaluation for mixed finite element methods. Computers and Structures 2001; 79:243-252.

13. Gajo A, Denzer R. Finite element modeling of saturated porous media at finite strains under dynamics conditions with compressible constituents. Internal Journal for Numerical Methods in Engineering 2011; 85:1705-1736.

14. Regueiro RA, Ebrahimi D. Implicit dynamic three-dimensional finite element analysis of an inelastic biphasic mixture at finite strain: part 1: application to a simple geomaterial. Computer Methods in Applied Mechanics and Engineering 2010; 199:2024-2029.

15. McGann CR, Arduino P, MacKenzie-Helnwein P. Stabilized single-point 4-node quadrilateral element for dynamic analysis of fluid saturated porous media. Acta Geotechnica 2012; 7:297-311.

16. Preisig M, Prevost JH. Stabilization procedures in coupled poromechanics problems: a critical assessment. International Journal for Numerical and Analyrical Methods in Geomechanics 2011; 35:1207-1225.

17. Truty A, Zimmermann T. Stabilized mixed finite element formulations for materially nonlinear partially saturated two-phase media. Computer Methods in Applied Mechanics and Engineering 2006; 195:1517-1546.

18. White JA, Borja RI. Stabilized low-order finite elements for coupled solid-deformation/fluid-diffusion and their application to fault zone transients. Computer Methods for Applied Mechanics and Engineering 2008; 197:4353-4366.

19. Levenston ME. Variationally derived 3-field finite element formulations for quasistatic poroelastic analysis of hydrated biological tissues. Computer Methods in Applied Mechanics and Engineering 1998; 156:231-246.

20. McTigue DF. Thermoelastic response of fluid-saturated porous rock. Journal of Geophysical Research 1986; 91(B9):9533-9542.

21. Scherer GW. Bending of gel beams: methods for characterizing elastic properties and permeaiblity. Journal of Non-crystalline Solids 1992; 142:18-35.

22. Roscoe KH, Schofield AN, Wroth CP. On the yielding of soils. Geotechnique 1958; 8:22-53.

23. Wriggers P, Reese S. A note on enhanced strain methods for large deformations. Computer Methods in Applied Mechanics and Engineering 1996; 135:201-209.

24. Borja RI, Alarcón E. A mathematical framework for finite strain elastoplastic consolidation part 1: balance laws, variational formulation and linearization. Computer Methods in Applied Mechanics and Engineering 1995; 122:145-171.

25. Sanavia L, Schrefler BA, Steinmann P. A formulation for an unsaturated porous medium undergoing large inelastic strains. Computational Mechanics 2002; 28:137-151.

26. Dormieux L, Kondo D, Ulm F-J. Microporomechanics. Wiley: New York, N.Y, 2006.

27. Rajagopal KR. An introduction to mixture theory. In Mathematical Theory in Fluid Mechanics, Galdi GP, Málek J, Necas (eds), Pitman Research Notes in Mathematics Series. Longman: London, 1996; 86-113.

28. Sun WC, Kuhn MR. A mulitscale DEM-LBM analysis on permeability evolutions inside a dilatant shear band 2012. Manuscript submitted to Acta Geotechnica.

29. Nur A, Byerlee JD. An exact effective stress law for elastic deformation of rock with fluids. Journal of Geophysical Research 1971; 76(26):6414-6419.

30. Rice JR, Cleary MP. Some basic stress diffusion solutions for fluid-saturated elastic porous media with compressible constituents. Reviews of Geophysics 1976; 14(2):227-241.

31. Mota A, Sun WC, Ostien JT, Foulk JW, Long KN. Lie-group interpolation and variational recovery for internal variables 2012. Manuscript submitted to Computational Mechanics.

32. de Souza Neto EA, Perić D, Owen DRJ. Computational Methods for Plasticity. Wiley: New York, N.Y, 2008.

33. Moran B, Ortiz M, Shih CF. Formulation of implicit finite element methods for multipicative finite deformation plasticity. International Journal for Numerical Methods in Engineering 1990; 29:483-514.

34. Simo JC, Taylor RL, Pister KS. Variational and projection methods for volume constraint in finite deformation elasto-plasticity. Computer Methods in Applied Mechanics and Engineering 1985; 51(1-3):177-208.

35. Broccardo M, Micheloni M, Krysl P. Assumed-deformation gradient finite elements with nodal integration for nearly incompressible large deformation analysis. International Journal for Numerical Methods in Engineering 2009; 78:1113-1134.

36. Harari I. Stability of semidiscrete formulation for parabolic problem at small time steps. Computer Methods in Applied Mechanics and Engineering 2004; 193:1491-1516.

37. Brezzi F, J. Pitkäranta J. On the stabilization of finite element approximation of the Stokes equations. In Efficient Solutions of Elliptic Systems, Vol. 10, Hackusch W (ed.), Notes on Numerical Fluid Mechanics. Vieweg Braunschweig: Germany, 1994; 11-17.

38. Bochev PB, Dohrmann CR. Stabilization of low-order mixed finite elements for the Stokes equations. SIAM Journal of Numerical Analysis 2006; 44(1):82-101. 
39. Burman E. Pressure projection stabilizations for Galerkin approximation of Stokes' and Darcy's problem. Numerical Methods for Partial Differential Equations 2008; 24:127-143.

40. Verfürth. Error estimates for a mixed finite element approximation of the Stokes problem. RAIRO Numerical Analysis 1984; 18:175-182.

41. Puso MA, Solberg J. A stabilized nodally integrated tetrahedral. International Journal for Numerical Methods in Engineering 2006; 67(6):841-867.

42. Puso MA, Chen JS, Zywicz E, Elmer W. Meshfree and infinite element nodal integration methods. International Journal for Numerical Methods in Engineering 2008; 74:416-446.

43. On the stability of dilatant hardening for saturated rock masses. Journal of Geophysical Research 1975; 80(11):1531-1536.

44. Rudnicki JW. Diffusive instabilities in dilating and compacting geomaterials. In Multiscale Deformation and Fracture in Materials and Structures, Chuang T-J, Rudnicki JW (eds). Kluwer Academic Publishers: Netherlands, 2000; 159-182.

45. Tezduyar T, Park Y. Discontinuity-capturing finite element formulations for nonlinear convection-diffusion-reaction equations. Computer Method in Applied Mechanics and Engineering 1986; 59(3):307-325.

46. Pawlowski RP, Phipps ET, Salinger AG. Automating embedded analysis capabilities and managing software complexity in multiphysics simulation, Part I: Template-based generic programming. Scientific Programming 2012; 20(2):197-219.

47. Pawlowski RP, Phipps ET, Salinger AG, Owen SJ, Siefert CM, Staten ML. Automating embedded analysis capabilities and managing software complexity in multiphysics simulation, Part II: Application to partial differential equations. Scientific Programming 2012; 20(3):327-345.

48. Heroux MA et al. An overview of the Trilinos project, ACM Transactions on Mathematical Software. Special Issue on the Advanced Computational Software 2005; 31(3):397-423.

49. Notz PK, Pawlowski RP. Graph-based Software Design for Managing Complexity and Enabling Concurrency in Multiphysics PDE Software. ACM Transactions on Mathematical Software 2012; 39(1):1:21.

50. Pawlowski RP. (Available from: http://trilinos.sandia.gov/packages/phalanx) [Accessed May 30, 2012].

51. Gay D, Phipps E. (Available from: http://trilinos.sandia.gov/packages/sacado) [Accessed May 30, 2012].

52. White JA, Borja RI. Block-preconditioned Newton-Krylov solvers for fully-coupled flow and geomechanics. Computational Geosciences 2011; 15:647-659.

53. Haider MA, Guilak F. Application of three-dimensional poroelastic BEM to modeling the biphasic mechanics of cell-matrix interactions in articular cartilage. Computer Methods in Applied Mechanics and Engineering 2007; 196:2999-3010.

54. (Available from: http://cubit.sandia.gov) [Accessed May 30, 2012].

55. Ogden RW. Non-linear Elastic Deformation. Dover Publication: Mineola, N.Y, 1997.

56. Scherer GW, Prevost JH, Wang ZH. Bending of a poroelastic beam with lateral diffusion. International Journal of Solids and Structures 2009; 46:3451-3462.

57. Johnson C. Numerical Solution of Partial Differential Equations by the Finite Element Method. Dover Publication: Mineola, N.Y, 2009.

58. Foster CD, Regueiro RA, Fossum AF, Borja RI. Implicit numerical integration of a three-invariant, isotropic/kinematic hardening cap plasticity model for geomaterials. Computer Methods in Applied Mechanics and Engineering; 194(50-52):5109-5138.

59. Sun WC. An unified method to predict diffuse and localized instabilities in sands. Geomechanics and Geoengineering. DOI: 10.1080/17486025.2012.695403. 